PNL-10378

$\mathrm{UC}$

$0 \partial \partial$

Peer Review of the Barselina Level 1 Probabilistic Safety Assessment of the Ignalina Nuclear Power Plant, Unit 2

\title{
SL McKay
}

GA Coles ${ }^{(a)}$

January 1995

Prepared for

the U.S. Agency for International Development and the U.S. Department of Energy under Contract DE-AC06-76RLO 1830

Pacific Northwest Laboratory Richland, Washington 99352

(a) Westinghouse Hanford Company

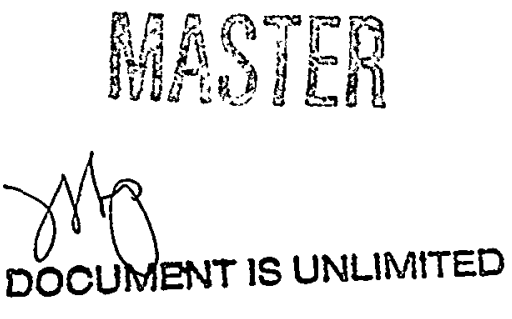





\section{DISCLAIMER}

This report was prepared as an account of work sponsored by an agency of the United States Government. Neither the United States Government nor any agency thereof, nor any of their employees, make any warranty, express or implied, or assumes any legal liability or responsibility for the accuracy, completeness, or usefulness of any information, apparatus, product, or process disclosed, or represents that its use would not infringe privately owned rights. Reference herein to any specific commercial product, process, or service by trade name, trademark, manufacturer, or otherwise does not necessarily constitute or imply its endorsement, recommendation, or favoring by the United States Government or any agency thereof. The views and opinions of authors expressed herein do not necessarily state or reflect those of the United States Government or any agency thereof. 


\section{DISCLAIMER}

Portions of this document may be illegible in electronic image products. Images are produced from the best available original document. 


\section{Executive Summary}

The Barselina Project is a Swedish-funded, cooperative effort among Lithuania, Russia and Sweden to transfer Western probabilistic safety assessment (PSA) methodology to the designers/operators of Ignalina Nuclear Power Plant (INPP). The overall goal is to use the PSA as a tool for assessing plant operational safety. The INPP is a two-unit, Former Soviet Union-designed nuclear facility located in Lithuania. The results of this PSA will ultimately be used to identify plant-specific improvements in system design and the conduct of facility operations, allowing improved operational safety. Pacific Northwest Laboratory (PNL) $)^{(a)}$ was asked to perform an independent expert peer review of the Barselina PSA. This report documents the findings of this review.

This review, financed with nuclear safety assistance funds through the U.S. Agency for International Development (USAID) and the U.S. Department of Energy (DOE), satisfies

- Task II of the PNL peer review of the Barselina project. The objective is to provide an independent, in-process examination of the Barselina Level 1 PSA of Ignalina Nuclear Power Plant, Unit 2. The review consisted of an investigation of the project documentation, interviews, and extensive discussions with the PSA staff during critical stages of the project. PNL assessed the readability, completeness, consistency, validity, and applicability of the PSA. The major aspects explored were its purpose, major assumptions, analysis/modeling, results, and interpretation. It was not within the scope of this review to perform plant walkdowns or to review material other than the PSA documentation.

PNL reviewers found the Barselina Level 1 PSA of INPP, Unit 2 to be a thorough, detailed, and scrutable assessment that advances the state of knowledge of the Ignalina plant and the Reactor Bolshoi Moschnosti Kanalynyi (RBMK) reactor plants. Because this PSA is a pioneering work, the understanding of this reactor through assessment processes can be expected to evolve with both the planned refinements to this assessment and the conduct of the upcoming Safety Analysis Review. As a natural product of this evolution, refinements in the quantification of core damage frequency and additional engineering insight could change the determination of the risk presented by this design. These refinements can profoundly impact the quantification of risk; therefore, this review endorses further PSA work based on the experience to date.

Assessment limitations identified through this peer review have been discussed with project management and documented within this report; they are presently in various stages of resolution. These shortcomings should not detract from the overall insight provided by this PSA project. This review encourages the careful consideration of all recommendations proposed for issue resolution. Additionally, it must be noted that the PSA methods applied during this project were developed for Western reactor systems. Accordingly, it is difficult to know whether the cultural issues of facility operation, management, and environment and the infrastructural differences of the RBMK are always accounted for the PSA processes. These considerations justify the continued assessment of this design with all available technologies.

(a) Pacific Northwest Laboratory is operated for the U.S. Department of Energy by Battelle Memorial Institute under Contract DE-ACO6-76RLO 1830. 


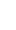




\section{Contents}

Executive Summary $\ldots \ldots \ldots \ldots \ldots \ldots \ldots \ldots \ldots \ldots \ldots \ldots \ldots \ldots \ldots \ldots$

1.0 Objectives and Scope of Review $\ldots \ldots \ldots \ldots \ldots \ldots \ldots \ldots \ldots \ldots \ldots \ldots \ldots$

2.0 Conclusions and Recommendations $\ldots \ldots \ldots \ldots \ldots \ldots \ldots \ldots \ldots \ldots \ldots \ldots$

3.0 Summary of Comments $\ldots \ldots \ldots \ldots \ldots \ldots \ldots \ldots \ldots \ldots \ldots \ldots \ldots \ldots \ldots \ldots$

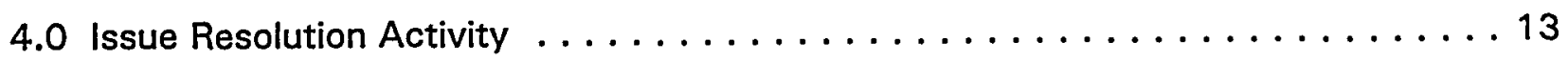

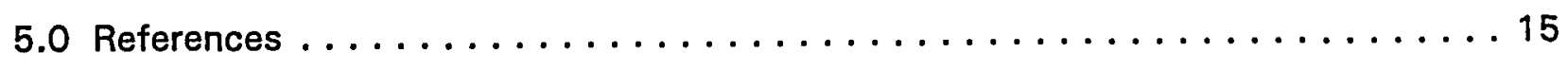

Appendix A: Phase II Comments and Responses . . . . . . . . . . . . . . A.1

Appendix B: Phase III Comments and Responses $\ldots \ldots \ldots \ldots \ldots \ldots \ldots \ldots$

\section{Tables}

3.1 Elements of a Complete and Readable PSA $\ldots \ldots \ldots \ldots \ldots \ldots$ 


\subsection{Introduction}

Pacific Northwest Laboratory (PNL) was invited to perform an independent expert peer review of the Barselina probabilistic safety assessment (PSA). The Barselina Project is a cooperative effort among Lithuania, Russia, and Sweden. Its aim is to transfer PSA methodology to Lithuania as a tool for managing reactor plant safety. A PSA was performed for the Ignalina reactor plant in Lithuania by distributing work among the three countries. The results of the PSA will be used to identify plant-specific improvements in system design and operating or maintenance procedures. This document reports the findings of PNL's review of the PSA.

This review, financed with nuclear safety assistance funds through the U.S. Agency for International Development (USAID) and the Department of Energy-(DOE), satisfies Task II of the Pacific Northwest Laboratory (PNL) peer review of the Barselina project. The objective is to provide an independent examination of the Barselina Level 1 PSA of Ignalina Nuclear Power Plant, Unit 2. This review is based on investigation of the project documentation and interviews with the PSA staff at different stages during the project. The review assesses the readability, completeness, consistency, validity, and applicability of the PSA. The major aspects we explored were its purpose, major assumptions, analysis/modeling, results, and interpretation. It is not within the scope of this review to perform plant walkdowns or review material other than the PSA documentation.

This review benefits from insights gained while performing the Hanford $N$ Reactor Level III Probabilistic Risk Assessment (Zentner et al. 1990) and external events analysis. The N Reactor was the only full-scale, graphite-moderated, pressurized water channel reactor that produced electricity in the United States, and it, like the Reactor Bolshoi Moschnosti Kanalynyi (RBMK) plants, presented unique challenges to system modeling techniques required by the PSA process.

This review was done in two stages and funded as two distinct tasks: The first, Task 1, reviewed the Barselina Phase II report; the second, Task 2, reviewed the more fully developed Barselina Phase III report.

The Phase II draft documentation was published in June 1993 and consisted of initiating event screening, accident sequence (event tree) and fault tree modeling, data collection, and preliminary sequence quantification using screening values for selected accidents. The peer review (Task 1) at this critical point included a comment and response period that provided important insight into the issues and their resolution that could be factored into the development process. These comments and responses can be found in Appendix $A$ of this report.

The Phase III report, published in June 1994, consists of finalized documentation, system modeling, final core damage accident sequence quantification, and the results. As with the Task 1, Phase II review, Task 2 activities provided for a comment and response period to.allow for issue clarification and resolution. These comments and responses are contained in Appendix $B$ of this report. 
The Barselina project will continue with the implementation of a Phase IV. This phase will treat outstanding risk-significant issues, some of which are developed from this peer review.

This review is somewhat different from others in that it documents both comments and responses and relates project intention with respect to issue resolution. Due to this relationship and because this is an in-process review, this review provides a quality assurance function. However, this is not the main purpose. The primary purpose is to provide independent expert peer review. Overall, this review summarizes the strengths and limitations of the Phase III work and identifies issues that need further analysis and that are, therefore, candidates for treatment in Phase IV.

Section 2 of this report provides conclusions and recommendations of comments. Section 3 provides a summary of the comments that are individually addressed in Appendix B. Section 4 provides a discussion of follow-up PSA activities that could be performed in the upcoming Phase IV effort of the Barselina project.

As stated previously, Appendixes A and B contain the detailed comments and responses from Phases $I I$ and III, respectively. The review comments are divided into six functional areas: 1) the overall scope and assumptions (O), 2) initiating events (I), 3) event tree models $(E), 4)$ data frequency and probability determination (D), 5) fault tree and system modeling and Human Reliability Analysis (HRA) (F), and 6) quantification and interpretation of the results $(R)$. 


\subsection{Conclusions and Recommendations}

The Barșelina Level 1 Probabilistic Safety Assessment (PSA) of Ignalina Nuclear Power Plant, Unit 2 is a thorough, detailed, and scrutable assessment. It advances the state of knowledge of the Ignalina plant and the Reactor Bolshoi Moschnosti Kanalynyi (RBMK) reactor plants in general. Because it is pioneering work, further evolution in understanding this reactor is expected through the assessment processes. In particular, upcoming work to develop a Safety Analysis Report (SAR) will add to the knowledge base of this design. Refinements in the quantification of core damage frequency and additional engineering insights could change the determination of risk presented by this facility. We endorse the plan to further this PSA work in Phase IV.

In many respects, this assessment is more comprehensive than other contemporary Level 1 PSAs. This review found it, the first full-scope PRA conducted on a formerSoviet-Union RBMK design; to be somewhat broader in scope than many other Level I PSAs. The PSA staff and management were found to be highly trained, knowledgeable, and competent practitioners of state-of-the-art assessments. And several aspects of the study deserve special commendation.

The difficult logistics of a multilateral study aside, the following general aspects of the PSA deserve special note: 1) the extensive common-cause analysis, including area event analysis; 2) the comprehensive multifunctional approach to initiating event identification; 3) the development of multiple core damage hazard states; and 4) development of a plant-specific database.

Some assessment limitations were also identified and are described below, but these should not detract from the overall insights gained from conducting this project. While we believe that these comments are important, it must be understood that the PSA methods applied by the Barselina project were developed for and applied to Western reactors. Accordingly, it is difficult to know if the operating, management, environmental, and infrastructure differences related to an RBMK are always accounted for in the PSA process. We noticed that certain changes in the plant operation and equipment had already been anticipated and credit taken in the risk assessment. Our concern is that, in time, the need for these changes will become obscured as the focus at the plant moves away from risk assessment to other business. There is some possibility this work will not be done, which adds to the uncertainty of the quantified risk.

The most significant limitations of this assessment involve

- Modeling the reactivity control system. The reactivity control system modeling is not fully developed and is not supported by adequate success criteria. Consequently, the reliability appears to be optimistic. 
- Lack of an uncertainty analysis. The availability of design-specific and archived information at Ignalina is limited, creating the potential for large data uncertainties. Large data and modeling uncertainties typically lead to large uncertainties in results, but this has not been addressed in this assessment. More plant-specific data analysis may be needed.

- Unavailability of deterministic analysis to support the various success criteria. Many areas of this assessment have been handicapped by the weakness of available analytical information and capability.

- Technical limitations of the assessment in capturing possible equipment and structural quality concerns and secondary effects such as pipe whip or steam on equipment or structures. Some aspects of the assessment, including plant-specific data development and area event analysis, begin to address the problem but not in its entirety. Risks due to external events such as earthquakes, flooding, and fires could be important but are not within the scope of this assessment. Fires and floods were treated, but only as internal events.

Issues of lesser significance include the following:

- Treatment of the group distribution header (GDH) blockage event. The importance of the GDH blockage event is somewhat obscured. If treated differently, the core damage frequency could be $1 \mathrm{E}-3 / \mathrm{yr}$, which is an order of magnitude higher than the total reported core damage frequency.

- Small loss-of-coolant accident (LOCA) frequencies. The small-break LOCA frequencies appear nonconservative due to the magnitude of small piping involved in this design.

- Instrument and control modeling. Instrument and control, with some exceptions, are not modeled in detail; loss of instrument power can be particularly important, but it is not considered in the assessment.

- One aspect of the Human Interaction Analysis approach. The weighing scheme used in the determination of error probabilities for human interactions may be nonconservative in certain cases. This scheme should accurately represent the operating culture.

- The scope of this assessment with respect to the plant operating mode. The scope of this assessment is limited to full-power events. Recent concern about low-power operational events has increased since the Chernobyl accident. The impact of this accident and the similarity of the designs mandate consideration of other-than-fullpower events.

We recommend resolution of the issues identified through this review. The relative importance of these issues has been discussed in this section; detailed discussions and potential resolutions are provided in the appendixes. Phase IV of the Barselina project will carry forward selected PSA development issues for potential resolution. The identified 
issues have been grouped into three methods for addressing resolution: 1) clear intention to do further PSA work, 2) plans to reassess certain issues during Phase IV after the SAR is complete, and 3) uncertainty development and application, to determine whether certain issues merit further expenditure of resources. This review endorses these commitments for resolution. 
. 


\subsection{Summary of Comments}

This section describes both the strengths and weaknesses of this Probabilistic Safety Assessment. The detailed comments supporting these determinations are found in Appendixes A and B for Phases II and III, respectively. The comments for Phase II (Appendix A) represent PNL Task 1 results, and the comments for Phase III (Appendix B) represent PNL Task 2 results.

Many of the concerns raised during the PNL Task 1 and 2 reviews involved issues already identified by the Barselina project staff. However, due to time constraints or other logistical reasons, they were not fully resolved. Because the project uses a phased approach, some issues will probably be carried on into the next phase of project work for resolution (see Section 4).

We found the Phase III analysis documentation to be detailed and comprehensive. In many respects, this analysis is more comprehensive than other contemporary Level 1 PSAs. This is the first full-scope Level 1 PSA performed for an RBMK, and this attention to completeness is warranted, because the design and operation of the RBMK are unique among commercial plants. This documentation has the qualities of a comprehensive and readable PSA as described in El-Bassioni et al. (1985) and itemized in Table 1.

Several aspects of this study deserve special commendation. Without providing credit for the difficult logistics of conducting a multilateral study, the following aspects of the study are noted:

- In general, the organization and readable quality of the PSA documentation is outstanding. It appears that all available pertinent and important information was documented as part of the PSA report.

- The assessment analysis of common-cause initiators is detailed and thorough and contributes to the comprehensive nature of the initiating event list.

- Inclusion of area event index analysis is innovative and beyond the scope of most Level 1 PSAs. This also contributes to the comprehensive nature of the initiating event list.

- In general, we support the multifunctional approach to development of an initiating event list. The unique design of this reactor demands the special effort made in this area.

- The Human Reliability Analysis (HRA) method developed by project staff is innovative and provides insights into understanding and addressing HRA risk-related issues. 
Table 1. Elements of a Complete and Readable PSA

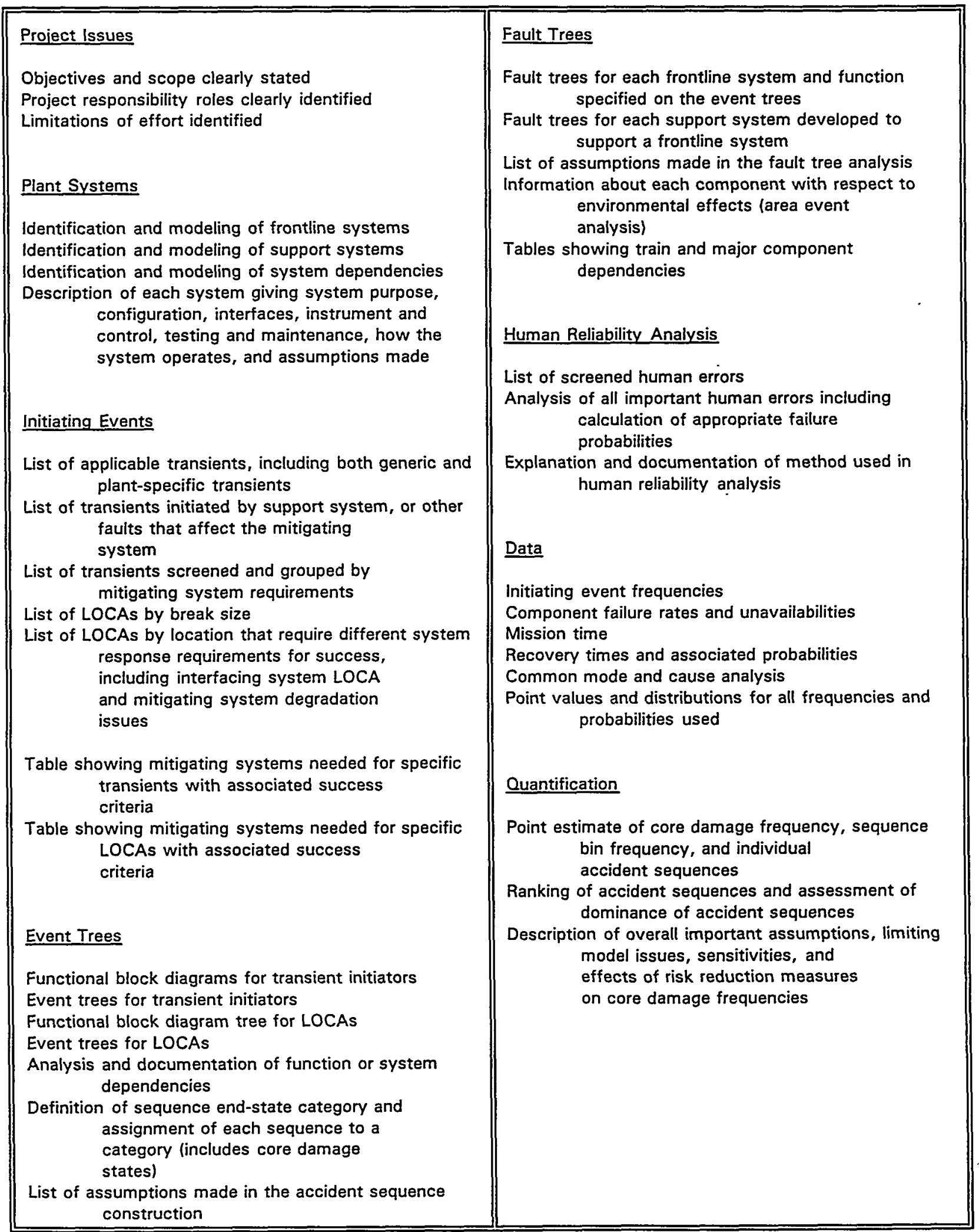


- The accident sequence analysis description format in Volume II, Section 4 of the PSA is, compared with many other PSAs, very comprehensive and detailed.

- The plant-specific database for the Ignalina Nuclear Power Plant provides a good foundation for understanding component failure rates and initiator frequencies at the plant.

- The event tree and fault trees are complex and provide enough detail to represent this plant. The entire model taken together is larger than those found in many contemporary PSAs. It contains about $50 \%$ more basic events than the $\mathrm{N}$ Reactor PSA. This approach might be described as a large-fault-tree-large-event-tree approach.

- The effort to differentiate core damage states is innovative and appropriate for this study. It leads to a much better understanding of the core damage frequency.

'The overall objective of this review demands an understanding of the assessment's composition and completeness. Supporting this objective required reviewing a large number of issues requiring subsequent examination and discussion. The majority of the issues raised during the PNL Task 1 (Phase II) comment period were addressed by project staff in their Phase III work. This was a natural evolution of the assessment process. Most issues raised during Task 1 are not summarized here, unless they were found to still be an issue during the review of the Phase III documentation. In some cases, the review comments affected the course of the Phase III analysis, allowing for issue resolution.

These are the most underdeveloped areas of this assessment:

- Modeling of the reactivity control system. The reactivity control system modeling is not fully developed and is not supported by adequate success criteria. Consequently, the reliability appears to be optimistic.

- Lacḱ of an uncertainty analysis. The availability of design-specific and archived information at Ignalina is limited, creating the potential for large data treatment uncertainty. Large data and modeling uncertainties typically lead to large uncertainty in results; however, this was not addressed in this PSA.

- Unavailability of deterministic analysis to support the various success criteria. Many areas of this assessment have been penalized by the weakness of available analytical information and capability.

- Technical limitations of the assessment in capturing possible equipment and structural quality concerns and secondary effects such as pipe whip or steam on equipment or structures. Some aspects of the assessment, such as plant-specific data development and area event analysis, begin to address the problem, but not in its entirety.

The following are highlights of issues that are described in more detail in Appendix B: 
Reactivity control reliability is optimistic, and models do not include common-cause events. Better technical bases and related success criteria are needed for local and general reactivity transients.

- No uncertainty analysis has been presented. At least a few dominant risk contributors appear to have very large uncertainties. An accurate determination of the uncertainties could potentially affect the useability of some of the PSA results.

- The deterministic analysis supporting the assumptions and technical bases is inadequate in certain areas. The amount and arrangement of piping flows tend to make the determination of success criteria more complex, enhancing the need for justification. As an example, discussions of success criteria in the report do not include reference to supporting technical documentation. This is the first reactor in the world for which PSA work has been accomplished ahead of SAR work. SAR documentation can typically provide some deterministic analysis applicable to PSA activities that is not available here.

- Structural and equipment quality and secondary effects are not fully accounted for. Dynamic effects such as pipe whip after break and steam affects on unqualified equipment is acknowledged but not included in the quantification of risk. The negative effects of certain construction and manufacturing practices are difficult to identify and are not well represented by current generic alpha factors for commoncause effects.

- $\quad$ LOCA frequencies, particularly small LOCA frequencies, might be nonconservative. This type of plant has orders of magnitude more small piping than Wester nuclear power plants, so more breaks might be expected. Data supporting the zero occurrence approach to determining LOCA frequencies are not compelling because of the lack of combined history.

- Instrument and control, with some exceptions, are not modeled in detail. Loss of instrument power could be particularly important; not separating critical loads has already proven to be an issue.

- The weighing scheme used in the determination of error probabilities for Human Interactions should be reevaluated, because it could be nonconservative in certain cases. Using the provided scheme, severe and important contributors to human error might have only a limited effect on calculating the error probability.

- The impact on the core damage frequency resulting from a group distribution header blockage event is somewhat obscured by its special treatment as a sensitivity. If this event were evaluated using the failure rates commonly used in Western PSAs, it would dominate the results (1E-3/yr).

- Non-full-power operating mode events are not included and are accurately represented as outside the defined scope of this analysis. Although this is a prerogative of the Barselina staff, consideration of non-full-power events could be critical to the risk quantification of this design. 
- Component reliability and parameters for initiator frequency distribution functions contain suspect determinations. These were developed from plant-specific data of limited scope. This is particularly true for events for which there have been few or no prior occurrences.

- No basic events quantitative importance analysis has been presented. Importance analysis would improve the useability and understanding of the results. For example, there are certain human errors contained in a large number of different sequences that would be identified as important by this analysis. Stated engineering improvements are difficult to evaluate without the screening process provided by the importance analysis.

- All human errors related to failure to restore after test or maintenance were assigned a probability of $1 \mathrm{E}-4$ with little discussion or justification. This appears to be nonconservative and optimistic. The same level of rigor was not applied here as was in assessing other human errors. 
$\cdot$ 


\subsection{Issue Resolution Activity}

All comments on the PNL Task 2 (Phase III) review have been presented to the Barselina staff. The comments are divided into six functional areas: 1) the overall scope and assumptions (O), 2) initiating events (I), 3) event tree models (E), 4) data frequency and probability determination (D), 5) fault tree and system modeling and Human Reliability Analysis (HRA) (F), and 6) quantification and interpretation of the results (R). Responses have been acknowledged and a potential resolution provided. Four types of responses were given: 1) There is a clear intention to do further PSA work in Phase IV of the Barselina project; 2) Certain issues will be reassessed during Phase IV, after the SAR is complete; 3) It is uncertain whether a certain issue merits further expenditure of resources; and 4) The basis is given for why no further assessment is needed for the stated issue.

The following responses are summarized here and contain designations (e.g., 01 , E5, F5) that refer to specific discussions in Appendix B, Phase III-Task 2 comments and response.

1. There is a clear intention to do further Phase IV PSA work:

- An uncertainty analysis is planned for the Phase IV activity. Probability distributions for both component reliability and initiator frequency will be reexamined. Better estimates of distribution parameters will be given to those values that are currently suspect (see comments 04, D1, D5, and R1).

- An importance analysis of basic events will be reported in Phase IV documentation. This analysis has actually already been performed (see comments 03 and R4).

- Reactivity transients and control models will be further developed. Common-cause, dependency, and area-event concerns will be incorporated. The technical bases for reactivity control success criteria (general and local reactivity transients) will be defined (comments 14, E2, F2, and R2).

- Certain area event and instrument and control modeling concerns will be addressed through the integration of the room dependency analysis with the quantification of core damage (comments 19 and F12).

- Recovery actions will be addressed as the plant develops formal procedures (see comment R3).

- A more developed comparison of the Ignalina PSA to the Barseback PSA will be included in Phase IV documentation (see comments 01 and D3).

- General plant technical descriptions will be developed and documented in an effort separate from Phase IV (see comment 02). 
2. There are plans to reassess the issue after the SAR work is done:

- Those issues affecting success criteria and important to the risk assessment will be identified and addressed in the SAR effort. The results will be addressed in Phase IV (see comment I7).

- If SAR information warrants evaluation of secondary effects (pipe whip or the effects of steam on unqualified equipment), it will integrated into the assessment activities of Phase IV (see comments E4 and E5).

3. It is uncertain whether the issue warrants further treatment:

- The weighing scheme used in the determination of error probabilities for human interactions does not adequately quantify impacting performance attributes (comment F6).

- The probability of properly restoring equipment after test or maintenance appears optimistic (comment D7).

- The possibility of unusual failure modes related to integrated circuits, particularly in the FASS and AZ- 1 is not recognized (comments 13 and F3).

- The steam drum separator rupture event could be treated as a sensitivity issue due to the failure frequency variance of provided evaluation methods (see comment 16).

- The accident frequency for certain sequences appears to be over-predicted by as much as a factor of 50 . This computer code manipulation error occurs when certain success branches have very high probabilities. This tends to skew the importance of certain sequences such as S1-1T.C2, No.2, which may not, in fact, be a dominant sequence. There could be other errors of similar nature (see comment R6).

4. No further assessment is needed:

- Editorial comments are acknowledged (see comments 11, 12, E1, F4, and F9).

- There was a misunderstanding on the part of the reviewer that was alleviated with further explanation (comments 15, F1, F7, F10, F11).

- The comment was acknowledged, but was not important enough to warrant change (D2, E3).

- Opinions vary with regard to the conservatism involved (see comments 18, D6, D4, F5, F6, O5). 


\subsection{References}

El-Bassioni, A., N. Z. Cho, N. Hangan, M. W. McCann Jr., J. O'Brien, I. A. Papazoglou, J. W. Reed, K. K. Shiu, T. Tiechmann, and R. W. Youngblood. 1985. PRA Review Manual. NUREG/CR-3485, Brookhaven National Laboratory for the U.S. Nuclear Regulatory Commission, Washington, D.C.

Zentner, M. D., J. K. Atkinson, P. A. Carlson, G. A. Coles, E. E. Leitz, S. E. Lindberg, T. B. Powers, and J. E. Kelly (SAIC). 1990. N Reactor Level 1 Probabilistic Risk Assessment: Final Report. WHC-EP-0322, Westinghouse Hanford Company, Richland, Washington. 
Appendix A

Phase II Comments and Responses 


\section{Appendix A}

\section{Phase II Comments and Responses}

\section{A.1 Overall Scope and Assumptions}

The following records comments and responses generated for Phase II of the Barselina project in February of 1994. Phase II was the initial phase of the Level 1 PSA for the Ignalina Unit 2 performed by the Barselina staff. This record consists of PNL review comments and the corresponding responses given by the project staff.

Comment 01:

Beyond design-basis accidents have been excluded from this PSA. For example, the rupture of the drum separator, as stated in Volume 1, is omitted from this assessment. This represents a departure from standard PSA protocol. The Russian (Polyakov/Shirersky) initiating event report, included in Volume 1 of the Phase II PSA documentation, specifically recommends inclusion of beyond-design-basis events. Beyond-design-basis events should be considered for inclusion in this assessment.

Response:

Beyond-design-basis accidents, including the drum separator rupture, will be included during Phase III of the project effort. Project personnel plan to use industry standard protocol with regard to the consideration of beyond-design-basis accidents.

\section{Comment 02:}

The scope of this assessment is limited to full-power events only. Pre-Chernobyl reactor PSAs typically assumed that the dominant risk was associated with fullpower operation. This assumption has been challenged ever since the core melt accident at Chernobyl, Unit 4, which was initiated at low power. The similarity of the Ignalina design to that of Chernobyl warrants consideration of other-than-fullpower events by the Barselina Project.

Independent peer reviews of the Level $1 \mathrm{~N}$ Reactor PSA by other DOE sponsored contractors criticized the project for not aggressively investigating non-full-power events. As a result of this concern, plans to model non-full-power events were under way at the termination of the N Reactor PSA project. (A full-scope, Level 3 PSA of the N Reactor [Zentner et al. 1990] was completed in 1990 using NUREG1150 (Sandia 1989) methodology.)

\section{A. 1}


Response:

The assessment will include only full-power operating events. Full-power operation is defined to be normal operating power down to 50\% of normal operating power. (The plant is administratively not allowed to operate under the $50 \%$ level). Project personnel recognize that the Chernobyl event did not occur at full operating power but believe that this type of event is no longer credible because of changes in the operating culture and design. Project personnel agree that justification of this position should be added to the Phase III documentation to clarify this concern.

This assessment includes only accidents that lead to offsite releases and affect the reactor core. Accidents involving the refueling machine will be added in Phase III; however, accidents involving the refueling machine that only affect the onsite worker and not the core will be excluded. Project personnel plan to provide further clarification for this rationale in the Phase III documentation.

\section{Comment 03:}

The mission time for most accident sequences appears to be 8 hours. Most industry standard PSAs employ a mission time of 24 hours. Mission times of less than 24 hours could result in overly optimistic reliability on long-term emergency core cooling system (ECCS) cooling. This would, in turn, lead to underpredicting the core damage frequency. Difficulties could arise when comparing this PSAs analysis with those that consistently use a mission time of 24 hours.

\section{Response:}

In Phase III, some accident sequences will possess a mission time up to 72 hours; however, these sequences are exceptions. In general, the mission time will be 8 hours. Earlier in the project, a 24-hour mission time was considered. In most scenarios, after 8 hours, there is a great deal of time and flexibility to recover plant systems. This allows use of an 8-hour mission a majority of the time without technical penalty to the assessment. Due to this and other practical factors, it is believed that the 8-hour mission time is adequate. Mission times of greater than 24 hours, and up to 72 hours, will be important in certain sequences, and these will be modeled accordingly.

\section{Comment 04:}

The PSA documentation does not provide a method to. account for issues that arise from philosophical differences related to the operation and management of Sovietdesigned facilities. Comparing this assessment with that of a Western facility should account for this difference. This issue will be of interest to outside peer reviewers and to the PSA community in general. A discussion of this accounting should be included in the final assessment documentation. 
Response:

As stated in the project documentation, the primary purpose of the Barselina project is to compare the Ignalina PSA with that of a Western facility. The Level 1 Ignalina PSA is to be compared with the Swedish PSA done on the Barseback facility. This comparison will be conducted within this Appendix and included in the results/ conclusions chapter of the Phase III documentation.

\section{Comment 05:}

A general description of the reactor core or reactor plant has not been included. Limited one-line diagrams and system descriptions have been provided. It is difficult to get a clear understanding of the overall facility, including system interfaces, from this material. The reactor is not described at all. A good technical description of the facility will enhance the readability of this report. Considering the RBMK's unique design, basic information concerning the reactor core should be included. System interface and dependency is extremely important to understanding and using this assessment. This dependency can be enhanced through the presentation and use of comprehensive system diagrams.

Response:

A general plant technical description will be included in the Phase III documentation. When the Phase II report was issued, there was no plant description available from the facility. An effort is being made to author a plant description by the Ignalina Safety Analysis Group and the Lithuanian Energy Institute (Almenas et al. 1994). These authors will provide the Barselina project with a specially formatted copy for inclusion in Ignalina PSA documentation.

Comment 06:

System descriptions could include a broad spectrum of information. As a minimum, the following should be considered for addition into the various system descriptions:

- A description of normal system operation including flows, temperatures, and pressures

- Applicable technical specifications

- A description of associated instrument and control circuit logic.

The RBMK is not a familiar design. To enhance the usability of this PSA, detailed system information should be developed and included as an integral part of PSA documentation. The N Reactor PSA provided detailed system descriptions (system notebooks) for each plant system important to the PSA. Each description, or notebook, was a substantial document in itself. 
Response:

More system information will be included in the Phase III documentation, although the project is limited by the amount and type of plant-specific information that has been archived. For example, until recently, there was no documentation equivalent to Western technical specifications. Information will be included as it becomes available. Phase III of the project will include a review by project personnel to examine the type of system information that will be incorporated into the assessment documentation.

\section{Comment 07:}

Recovery actions have not been included. System flexibility and reliance on operator action suggest consideration of recovery actions. Inclusion of recovery action could significantly alter the final core damage frequency. Applicable recovery action will allow a clearer understanding of accident mitigation activities and their importance.

Response:

A limited number of recovery actions will be included in the Phase III documentation. These will be limited to the manual restart of pumps and restoration of the electrical power grid. Due to the lack of formal emergency procedures, potential recovery actions will not be considered in the Phase III accident sequences. This can profoundly impact the quantification of the potential risk associated with a given sequence. Any analysis of recovery actions that is provided will be included as a Type 5 Human Reliability Analysis (HRA).

\section{Comment 08:}

A number of area-dependent effects have not been considered in determining equipment availability, including the possible effects of steam, high temperature, or LOCAs on important equipment. Some equipment required for accident mitigation may not be environmentally qualified to operate in the adverse environment created by postulated accidents. The failure to model area-dependent effects as basic events in the fault trees will preclude the evaluation of their significance with regard to accident progression. The documentation does not discuss equipment qualification and related environmental impact. Such area-dependent effects should be modeled as basic events in the fault trees.

Response:

Location-specific effects such as fire, flood, and missiles are being addressed in Phase III. Facility walkdowns have been performed to provide for this concern. Project personnel have agreed that the effect of steam should also be addressed in Phase III to allow the impact on key equipment, not qualified to operate in a steam environment, to be evaluated. 


\section{A.2 Initiating Events}

\section{Comment 11:}

Industry standard methods, employed by this assessment for determining the initiating events, may not identify all events applicable to this design. Additional analysis of mechanistically possible initiating events will be necessary. Failure to determine all credible events will profoundly impact the ability of the assessment to identify the dominant risk contributors.

This issue was raised during the independent peer review of the N Reactor PSA. Similar to Ignalina, $N$ Reactor has a unique design. One beneficial technique employed by Los Alamos National Laboratory in their examination of the $\mathrm{N}$ Reactor was a master logic diagram (MLD).

The MLD technique is described in the NUREG Probabilistic Risk Assessment Procedures Guide (Hickman et al. 1983) as a way to address the issue of completeness in identifying initiating events.

\section{Response:}

The issue of identification and classification of initiating events is being addressed. The completeness of initiating event identification will be discussed in the Phase III report. Project personnel will consider the applicability of using the MLD process in determining potential initiating events.

\section{Comment 12:}

Only LOCAs and a number of transient events (loss of offsite power, loss of main feedwater, normal SCRAMs, turbine trip, and technological channel block) are modeled at this time. The scope of the initiating event list should be expanded. The following events are examples of industry standard events that may warrant consideration.

- Internal flooding lleaks from large water lines can degrade motor-control centers)

- Internal fires (particularly those that might be started by equipment failure, especially equipment that contributes to core cooling)

- Interfacing LOCA

- Loss of support systems (such as service water)

- LOCAs that stagnate flow may justify the consideration of flow stagnation events. (Partial pipe breaks on the inlet side of the reactor could stagnate flow to a portion of the core under certain circumstances. This could occur 
in situations when the reactor needs to be cooled by backflow. Stagnation could occur when backflow is restricted due to hydraulic pressure in the forward direction, because the pipe failure is not a guillotine break.)

- $\quad$ Failure of the BRU-K or BRU-B relief valves to re-close (failure of the main relief valves (MRVs) to reclose is listed as a LOCA contributor)

- Leak of the main circulating pump seals (depending on how the system is configured, these may represent some interdependency that would require consideration)

- Loss of heating, ventilation, and air conditioning (HVAC) (could have a location-dependent effect)

- Inadvertent ECCS actuation

- Loss of deaerator level.

Response:

The following consideration will be given to the various issues identified in the area of initiating events. About 15 new initiators are being added in Phase III, including:

- Loss of deaerator pressure control

- Loss of drum separator level control

- $\quad$ ECCS header rupture

- GDH blockage

- Common-cause initiators.

The common-cause initiators include loss of the service water (SW) system, electrical bus failure, and area event initiators. Area event initiators are locationdependent events referred to in Comment 08. The plant was divided into approximately 10 areas and evaluated with respect to fires, floods, and missiles. These become the area event initiators. It is assumed that an area event initiator fails the functions of systems located in the area considered.

Some interfacing LOCAs (but not all) are included as part of the Zone 4 and 5 LOCAs. Breaks in the ECCS header will be included in the Phase III report. The interfacing LOCA at Ignalina does not have the same implications as other reactors for two reasons: 1) Interfacing systems are rated for the same pressure; 2) The Ignalina reactor does not have a containment vessel.

A certain event description given in Volume 1 of the Phase II report discusses a stagnation event. It is assumed that when a break occurs in a certain location, stagnation of flow will occur $10 \%$ of the time. It is assumed, however, that due to the transient nature of the system during a LOCA, flow stagnation will exist for a limited period of time only: This time frame precludes fuel damage. 
The BRU-K relief valves, which are intended for primary circuit over-pressure, can be isolated. This isolation capability precludes their consideration as an initiator. The BRU-B relief valves are intended for the same purpose but are located on the same lines as the MRVs. These dump steam to the Accident Localization System (ALS) pools. These will be included in Phase III as contributors to a medium LOCA.

The loss of main circulating pump seal is not explicitly included. Project personnel plan to consider this issue for inclusion.

The effect of the loss of heating, ventilation, and air conditioning (HVAC) is being investigated and will be addressed at some level of detail in the Phase III report.

Inadvertent ECCS actuation is described in the event description of ECCS in Volume 1 as an event of no significance. This will be addressed in the Phase III documentation.

Comment 13:

Event trees, which contain sequences initiated by primary circuit blockage (Volume 9), only consider three or fewer technical channel failures. Blockage of larger lines, such as the group distribution header (GDH), could be an important accident contributor and should be considered by the assessment. Loss of flow in a part or all of the primary cooling system should be considered for inclusion in the initiator category.

As with N Reactor, the RBMK design offers the potential for damage associated with a discreet compartment of the core. Blockage of a single GDH would likely lead to such an event.

Response:

Blockage of the GDH will be added as an initiator in Phase III. This initiator could be important if there is difficulty recognizing a GDH rupture from instrumentation. A manual trip must be performed quickly; auto-trip of ECCS would be too late. Gate or check valve failures will be considered as contributors to this blockage event. 


\section{Comment 14:}

Using the screening criteria described in Volume 1, Section 3, Appendix 2 is not clear. Explanation of the screening methodology will be required in the final documentation. Understanding the initiating event screening will enhance the credibility of the assessment. Specifically, the roles of A through K classification and $Y / N$ designations used in each of the nine tables in Appendixes 1 and 2 are significant and should be understood.

\section{Response:}

This criterion is described in Volume 1, Section 3.4.1, and in Table 1. The yes/no correlates to $A$ through $K$ in Table 1. These help categorize initiating events.

\section{Comment 15:}

At this writing, screening tables found in Appendix 2 of Section 3 do not clearly demonstrate consideration of each of the initiating events found in the Section 3.0 appendixes. For example, hydrogen buildup in the CPS cooling circuit is found in Appendix 2, taken from the RBMK-1500 Technical Safety Report. Screening tables are not presently filled out. The completeness of the screening tables needs to be assessed prior to issuing the final report documentation.

\section{Response:}

The initiating event screening tables will be added to, and all tables will be revised and completed in Phase III.

\section{Comment 16:}

Initiating events associated with the ECCS headers have not been included at this point. There are two break locations of interest: 1) a pipe break in the ECCS header downstream of ECCS check valve(s) and 2) a pipe break in the ECCS header or interfacing system upstream of the ECCS check valve(s). The second break location is more significant if it occurs in conjunction with failure of the certain ECCS header check valve(s) to remain closed. Initiating events associated with the ECCS headers should be included as a part of the final assessment.

Response:

ECCS header rupture is being added. The possibility of a pipe break upstream of a failed ECCS check valve will be investigated among the issues related to GDH rupture. The need for inclusion will be evaluated during an upcoming meeting with the plant personnel. 


\section{Comment 17:}

Reactivity transient initiators have not been included in the PSA at this writing. As with other RBMK reactors, the Ignalina reactor has a positive void coefficient. This feature was a contributor to the catastrophic accident that occurred at Chernobyl. Unique design features such as the positive void coefficient need to be evaluated with regard to their applicability as reactivity initiators. Reactivity transients could be bounded by worst-case success criteria for worst-case reactivity insertion. The frequency of the bounding case could be conservatively calculated based on the sum of all reactivity transients.

Response:

The project has received documentation related to reactivity initiators from $R B M K$ specialists. This information and a structure such as an MLD (see comment 11) will provide a method to address this issue. After the range of initiators is identified, groupings can be determined and bounding cases set up. Three important types of activity perturbations will be included:
- Slow-large
- Fast-large
- Beyond design-basis.

\section{Comment 18:}

Limited data can lead to large uncertainties in calculation of initiator frequencies. Volume 5, Table 4.1 shows that initiating event frequencies will be calculated with a Bayesian update technique using plant-specific data. This volume also indicates that only a few years of operating experience can be collected. A strategy to address large uncertainties is not described. The treatment of data needs to be stated in the final assessment documentation.

\section{Response:}

Uncertainty distribution for initiating events will be developed in the same way as component failures. If log normal distributions are used, the corresponding error factors will be between 2 and 10; however, it is not clear that log normal distributions will necessarily be used. A comparison to Swedish generic failure rates will be made. Outliers will be treated in the same manner. The uncertainty analysis will be completed in Phase III.

\section{Comment 19:}

The common-cause initiators referred to in the Ignalina PSA are important and may require special attention. The common-cause effect can represent an important potential common-cause initiator for the RBMK design due to independencies. Such was the case in the N Reactor PSA, so a special effort was made to evaluate cutsets for common-cause effects that could have been overlooked. 
Response:

Common-cause initiators are addressed in detail in Phase III, including

- Common-cause failure and effect analysis

- Common-cause initiator screening

- Common-cause impact analysis to support alpha factors

- Dependent-event analysis

- Area-event analysis.

Accordingly, the treatment of common cause is done on several levels. Good plantspecific system-level data exist that can be used to generate alpha factors lalpha factors, which are event-based, will be easier to generate with the available data than beta factors, which are component-based). Dependent-event analysis can be used to address common cause between initiator and mitigator.

\section{A.3 Event Tree Models/Accident Sequences}

\section{Comment E1:}

The unique design of the Ignalina Reactor has a significant impact on core hazard states. Its channel reactor design allows the potential for partial core damage states. This uniqueness will need to be addressed in the final PSA documentation.

Like Ignalina, the $\mathrm{N}$ Reactor was a multichannel reactor. Both reactor cores consist of coolant channels that are somewhat insulated from each other by graphite moderator blocks. This multichannel feature divides the reactor into coolant compartments.

A significant portion of the core melt frequency reported in the N Reactor PSA was associated with far less than the full core. Due to the multichannel feature, the major contributor to the core damage frequency was loss of cooling to approximately one-sixteenth of the core.

The major compartments that appear to exist in the Ignalina reactor plant are a technological channel, channels associated with a single GDH, and channels associated with half of the reactor. Within these compartments there may be groups of channels that operate at higher power and, therefore, are more susceptible to failure by certain ECCS cooling criteria. This compartment concept was presented to project personnel during earlier communication and is provided with actual hazard state constructs in Appendix C.

Response:

A new development for core hazard states is being prepared for Phase III. The new elements of this construct are shown in Table E1. This model contains certain 
elements offered by PNL in earlier communication. The core cooling effectiveness lone tube, one GDH, half core, full core), shown as column headings, illustrate this. It may be necessary to collapse these groupings into a more manageable number later. It is not clear whether a Level 2 PSA will be performed. These hazard core states may be particularly helpful, because this concept is applicable to Level 2 development work.

Comment E2:

Additional branch points should be considered if core hazard states are to be further differentiated. For example, a LOCA in Zone 1 followed by failure of short-term ECCS is not presently, but could be, further differentiated. The subsequent failure of intermediate- or long-term cooling could lead to differing end-states. This differentiation requires adding new accident sequences.

Response:

Project personnel agree that it may be appropriate to add these new branches, particularly for cases such as the one cited. There was, however, some reluctance to add more branches, because the event trees are already complex. These issues will be addressed in some manner in the Phase III documentation.

\section{Comment E3:}

The ECCS header and associated check valves are not modeled in the fault trees and are, therefore, missing from accident sequence cutsets. These failures may represent important risk contributors and should be evaluated.

At $N$ Reactor, there are ECCS header check valves similar to those at Ignalina. If they failed to open, they would represent a major contributor to core damage. The Ignalina plant has a more complicated ECCS header arrangement. There are three ECCS headers for each GDH. Given the large number of check valves involved, failure of one or more of these valves could be important for certain scenarios and should be evaluated.

\section{Response:}

A new primary circuit analysis section will be added to Volume 2, Section 5.17. The primary circuit was not recognized as an important system until recently. This system may be added as a black box event in the fault trees for the Phase III effort. Project personnel will investigate whether to include the failure of this system as a top event. Its addition as a top event would require significant modification of the event trees. 


\section{Comment E4:}

The quantification process, including the process of linking top events, is not described. Information concerning this methodology and model manipulations should be included. We assume that a large-fault-tree approach is being used.

\section{Response:}

This project employs the "large-fault-tree approach, "meaning that fault tree top events are explicitly linked to produce accident sequences. Although the "largefault-tree approach" is employed, the event trees remain quite complex. This reflects the complexity of the plant. In the Phase III effort, a large amount of material will be added to Volume 3 including project management, general plant detail, and methodology description. The method description section will provide a discussion of quantification, including a technical description of the PSA computer code Risk Spectrum.

\section{Comment E5:}

Certain transfer gates shown in the Volume 7 definition of the functional top events cannot be found in the referenced volume or in the fault trees (Volume 6). The gates that start with the prefix "FE" appear to be missing. These apparently represent the combination logic for linking certain portions of the fault tree and need to be identified in the report documentation.

\section{Response:}

Transfer gates with the prefix "FE" referred to in Volume 7 are defined in Volume 4 , last section.

Comment E6:

One fault tree model is provided to represent both the intermediate- and long-term ECCS cooling systems. It is not clear how this is possible. Immediate ECCS cooling operates over a 1-hour mission time, and long-term ECCS cooling operates over an 8-hour mission time. This potentially precludes the use of a single fault tree model.

Response:

The same section of fault tree can be used for intermediate- and long-term cooling when the only difference is the mission time (1 hour versus 8 hours). This approach is possible using the PSA code Risk Spectrum. If the same basic event appears more than once in a cutset with different mission times, some events are still subsumed. 


\section{Comment E7:}

None of the accident sequences modeled in the LOCA event trees appear to address a pipe break in an interfacing system combined with boundary valve failure. The information provided indicates that interfacing system piping is qualified to the same degree as the pressure boundary piping. Depending on the system pressures, failure of a boundary valve could be masked. If the lost inventory does not flow to the ALS pools, the inventory is nonrecoverable. This issue should be addressed in the evaluation of long-term ECCS.

Response:

Certain interfacing LOCA initiating events are included as Zone 4 and 5 LOCAs. Failure of boundary valves is neglected in associated accident sequences. Failure to isolate the leak is included and will have the same effect. Long-term (72 hours) ECCS makeup is required when Zone 4 or 5 initiated accidents are combined with failure to isolate, due to the external loss of inventory. The purification and cooling system is required for this long-term cooling. Due to issues identified by this PSA, the Ignalina plant is presently being modified to provide adequate long-term cooling in such events. This plant modification will mandate a modeling change, which is presently deferred.

\section{A.4 Fault Tree Modeling}

Comment F1:

The emergency diesel fault tree does not contain support system development. Omission of support system development may result in failure to identify important failure modes or interdependencies. For example, the following developments are omitted: starting air, DC starting circuits, and fuel oil.

$N$ Reactor interdependencies were found to exist in the DC starting circuits. As with the DC support system, the fuel oil system may be a good candidate for further development due to the potential for common-cause failures.

Response:

Project personnel are not planning to model diesel support systems. They agree that it is important to investigate these systems for interdependencies. The starting air, starting DC circuits, and, to a certain degree, the fuel oil systems are separate and independent for each diesel. Accordingly, the failures related to these support roles should be reflected in the diesel-failure-to-start-and-run data. Project personnel will evaluate the fuel oil and jacket water systems for their potential for commoncause failures. 


\section{Comment F2:}

Basic events related to testing, maintenance, and human interactions described in Volume 2 of the PSA are not found in the fault trees. For example, basic event AFWSRL02515_VM3M, "Misalignment of closed valve RLO2S15 after test or maintenance," is mentioned on page 6 of Section 5.3 of Volume 2. However, it is not found on page AFWSP1A of the Auxiliary Feedwater fault tree in Volume 6.

Reactor PSA tree models typically contain a number of "Failure to restore after test or maintenance" errors. Treatment of these errors should be included as part of the fault trees.

Response:

The basic events from Volume 2, such as the one cited, contain the wrong basic event text. Text that reads, "Misalignment of closed valve after test and maintenance," should actually read, "Valve out of service due to test and maintenance." This can be verified from the specific basic event nomenclature associated with that basic event. The nomenclature, per typical PSA practice, defines the failure type. Misalignment errors are actually covered in the fault by basic events that read, "Valve erroneously closed." Out-of-service unavailabilities are not presently contained in the fault trees because they are being reworked. These unavailabilities will be reintroduced at the train level rather than the component level. New procedures being used at the Ignalina facility now control the unavailability at the train level.

Basic events related to misalignment and human interactions described in Volume 2 of the PSA that are not found in the fault tree will be incorporated and correctly worded in the Phase III report. Out-of-service issues due to testing and maintenance will also be addressed in Phase III.

\section{Comment F3:}

At this point, the PSA documentation does not include a fault tree or system description of the nitrogen system. This system appears to be important, because it is critical for ECCS short-term cooling. If it fails undetected, it may impact the ability of the ECCS to operate on demand. Consideration should be given to modeling the nitrogen system.

Response:

There are no plans to model the nitrogen system. The failure of the nitrogen system at the time of an accident is of no consequence if the pressure and volume of nitrogen in the head space of the ECCS pressurized tanks is adequate. Nitrogen system unavailability may contribute to the functional loss of driving pressure during a short-term ECCS demand; project personnel agree to investigate this concern. 


\section{Comment F4:}

Loss of power to service water (SW) pumps such as QF4ODO1 is not included in the fault tree model shown in Volume 6. This appears to be a critical omission due to the interdependence of SW to other important systems.

Response:

In the fault tree, the omission of loss of electrical power to the SW pumps is a logical loop cut. There are 12 to 18 similar cases in the fault trees. Project personnel acknowledge that, in the fault tree, the transfer gate connecting electrical power support cannot simply be omitted, because loss of electrical power needs to be accounted for. The starting circuits on the diesel also have similar logical loops. When starting the emergency diesels in absence of ac power, the diesel controller eventually (within a few seconds) has to bootstrap from electrical power produced. In Phase III, project personnel plan to perform logical loop cuts in a manner that does not exclude certain failure modes. They are considering employing a method described to them from the N Reactor PSA.

\section{Comment F5:}

Depending on the design, flow meters can represent flow blockage potential. The type of flow meter installed at Ignalina is not described in the system documentation, and its potential for flow blockage needs to be evaluated. This potential was evaluated for the $\mathrm{N}$ Reactor because, similar to the Ignalina plant, flow measuring instrumentation exists at the inlet of each process tube (technological channel).

\section{Response:}

It is not believed that the flow meters used at the Ignalina plant can fail in a way to block flow. Drawings provided by reactor plant personnel indicate that flow is measured by counting rotations of a spinning sphere. Evidence in the drawing is helpful but not conclusive. Project personnel have not been provided a detailed technical description of the flow measuring device to assist in this evaluation. Further consideration of this issue will be provided during the Phase III work.

\section{Comment F6:}

The basis for assuming that one ECCS and auxiliary feedwater (AFW) pump is always unavailable (out of service) is not clear. We assume that it was done to conservatively address test and maintenance out-of-service intervals. These unavailabilities are not found in the fault trees. The basis for this unavailability needs to be provided in the final assessment documentation. 
Response:

The basis for ECCS and AWS unavailability has changed. As stated in Comment F2, new plant out-of-service procedures are in place. The procedures are more restrictive than past practices and are instituted at the train level. Basic events that represent unavailability due to test or maintenance will be added to the fault trees in Phase III.

\section{Comment F7:}

Nearly all instrument and control systems are modeled with single basic events lloss of signal). The instrument and control systems should be modeled in more detail. For example, the deaerator level control is acknowledged as a very important system and should be considered for modeling.

The N Reactor PSA identified an important interdependency in the instrumentation for the primary system controllers. This interdependency. was discovered as a result of control system modeling. A single component failure was identified that could fail all power to the primary system controllers. This failure results in the loss of all associated controllers, which produces a severe system transient requiring ECCS recovery.

\section{Response:}

The Phase III report will include fault tree modeling of the drum separator level controller, deaerator pressure controller, actuation and control system (ACSS), reactor control and protection system, and possibly some local instrument and control systems. This, in turn, may require more dc power modeling.

Comment F8:

Cooling of the ALS pools during long-term ECCS cooling is not modeled as a support system. PSA documentation states that this failure could lead to ECCS pump cavitation. This seems to introduce another support system dependency. System modeling to allow evaluation for interdependency should be considered.

\section{Response:}

The dependency of ECCS makeup on cooling the ALS pools will be included in Phase III on a sequence-by-sequence basis. In LOCA scenarios, a substantial amount of inventory is being supplied from the auxiliary feedwater system, which mixes with the hot ALS pool water. For these cases, ALS pool cooling is not an issue. In transient scenarios ECCS makeup is also not an issue. For this reason, the cooling of the ALS pool was included in earlier project models but subsequently taken out. 


\section{Comment F9:}

Fault trees for the reactivity control system do not exist at this authoring. The reported reliability (1E-10) seems high compared with other systems. A fault tree for the reactivity control system needs to be developed to evaluate its reliability, and the reliability provided needs justification that should be included in the final assessment documentation.

Industry PSAs typically report reactivity control system reliabilities much lower than 1E-10. These reliabilities include credit for borated water as a backup system. The $N$ Reactor PSA reported values in the $1 E-7$ range. At $N$ Reactor, the control rods were backed up by boron balls; the Ignalina plant has neither a borated water nor a borated ball backup system. So it is not clear why the reliability of the Ignalina reactivity control system should be so high. In the N Reactor PSA, common-cause failure of certain sets of relays dominated the calculated failure of the reactor trip. system.

\section{Response:}

Project personnel have not received the Reactor Control and Protection fault trees from support personnel in Moscow yet. There are plans to add Alpha commoncause factors to the fault tree models when they are received. It is not clear whether Alpha factors will be introduced to the relays, power supplies, or both. In any event, the reliability of the system will be reduced as appropriate.

\section{Comment F10:}

Fault tree modeling of the HVAC system is not included at this point. In the absence of any other hydrogen mitigation systems, the operation of the HVAC system might be important to the progression of an accident after fuel damage has occurred. Consideration should be given to developing a fault tree for the HVAC system.

\section{Response:}

The HVAC system will be modeled both as an initiator (see Comment /3) and as a mitigator. However, it will not be modeled in detail because of time constraints. It will be modeled as a "black box" in Phase III. It is believed that failure of HVAC may be particularly important as a common-cause failure of the Reactor Control and Protection System (see Comment F9).

\section{Comment F11:}

Accident progression mitigation features such as gas holdup in the ALS tower have not been modeled at this point. Depending on the design intent of the ALS tower, modeling may be warranted. Consideration should be given to modeling the ALS tower and other systems that have an effect on accident progression or containment of hazardous material.

\section{A.17}


Response:

Modeling the cited system and other systems important to confinement of the core damage and accident progression issues is not within the scope of this analysis. There are no plans to do so during the Level 1 effort.

\section{A.5 Data}

\section{Comment D1:}

The basis for the number of demands or exposure times is not included in the PSA documentation at this point. Technical specification requirements for out-of-service times should be described. The basis for exposure times should be included in the final assessment documentation.

\section{Response:}

Discussion of mission times will be added to the system descriptions in Volume 2 in the section entitled "Restricted Operation." Exposure times will be taken from recently written procedures. Technical material from documentation of the Risk Spectrum code will be added to Volume 3, Appendix A in Phase III to describe calculational methods and files related to mission times.

\section{Comment D2:}

The justification for the frequency $1.0 \mathrm{E}-10 / \mathrm{yr}$ for rupture of steam drum lines to condensers or relief valve failure has not been included at the time of this writing. The justification for this frequency should be included in the final assessment documentation.

\section{Response:}

Comment is under consideration.

\section{Comment D3:}

Analytical work on data has not been provided in Phase II documentation. Use of the screening values, which appear to be the extent of the data work so far, require little documentation. The uniqueness of this assessment will mandate the documentation of data treatment methodology.

\section{Response:}

Uncertainty analysis and supporting discussion will be provided in Phase III. Completed plant-specific data have not been available to the project team at this time. 


\section{Comment D4:}

Screening valves based on Western/Swedish PSAs are used without discussion of applicability to-RBMK design. Screening values may or may not be conservative because of factors including economic hardship, maintenance and operation philosophy, and manufacturing processes.

Response:

The Phase III report will compare plant-specific data with Swedish generic data and with screening values. A discussion of these comparisons will be included, and outliers will be addressed.

\section{Comment D5:}

Certain accident cutsets could be screened out before plant-specific data are applied. The plans described in Volume 5 use Bayesian updating and seem appropriate, and the screening data appear to be conservative. However, it is possible to screen out cutsets before more accurate values are applied. This would happen if the plant-specific values were actually higher than the screening values (see Comment D7).

Response:

Although the data are referred to as screening data, no sequences will be eliminated as a result of quantification using screening values. The truncation level for quantification is set at 1E-9, which is comparable with many other PSAs. The screening values are more conservative than expected values; therefore, information should not be lost due to the truncation level.

Comment D6:

The summary of dominant contributors found in Volume 1, Section 2.5.2, Tables $V$ and VI could change when screening values are replaced.

Response:

The dominant risk contributors will sort differently in the Phase III report. It is believed that the incompleteness of the Phase II work will contribute to this difference more than any change in the data values.

\section{Comment D7:}

The medium LOCA requires operator action to identify, access, and isolate the break within two minutes. Even the 0.1 probability assigned to this human error could be nonconservative. Operational data indicate that an operator with little time to identify and carry out the corrective actions will err. Such errors, due to limited response time available to identify the fault, might actually aggravate the situation. 
Using Dougherty (1988), an analyst could take very little or no credit for success in the cited case. HRA screening values are not very conservative when compared with human reliability probabilities using Swain and Gutmann (1983). Justification for the HRA values used should be provided in the final assessment documentation.

Response:

As described in Comment D5, the screening values are meaningless. The HRA will be described in great detail in Volume 3, Appendix $C$, as will the exact methodology. It will not be a prescribed methodology such as Swain and Gutmann's (1983) handbook on HRA, but a generic analysis using various sources will be provided.

Comment D8:

At this point the fault trees contain few human errors, in spite of the fact that the plant operator is an important mitigating factor in many accident scenarios. We believe human reliability will be critical to this PSA. HRA should be included as an integral part of this PSA.

Response:

It is true that HRA will be an important part of this PSA. The HRA will be performed at the functional event level rather than at the basic event level, because of the phase-mission aspect of the accident sequences (i.e., the point in time that the action is taken with respect to the accident sequence is critical).

Comment D9:

At this point, common-cause analysis and HRA are not fully developed. Development using plant-specific data analysis will be important, as acknowledged in this PSA documentation. Common-cause and human error failures may be closely coupled for certain events.

Response:

Common-cause effects will be incorporated depending on the type of human error being evaluated. There are fives types of human error models that could be included:

- Latent errors such as valve misalignments

- Initiators

- Response actions to accident conditions (errors of omission)

- Errors of commission

- Recovery actions.

In Phase III, latent errors will be addressed at the train level, as described in Comment F2. Treatment at this level allows consideration of common effects. The 
human error contribution to initiating events will be reflected in the data. A full treatment of common-cause initiators is being performed. Response action will be analyzed in detail and attached to the model at the functional top event level as described in Comment D8. Errors of commission are not addressed. Recovery actions are being addressed on a the limited basis as described in Comment 07.

\section{A.6 Quantification/Results}

\section{Comment R1:}

Some of the accident sequences result in illogical cutsets. These cutsets contain an event that is shown both as a success and as a failure in the same cutset. For example, in Event Tree A-1.C, sequence \#15, top event V1.2 is shown as successful. However, cutsets listed in Volume 8 show failure, ECCS_RIGHT_ST_F, as a contributor to sequence \#15; and Volume 4 shows failure, ECCS_RIḠHT_ST_F, is a contributor to failure of top event $V 1.2$. This appears to be a contradiction and has the potential to be a systemic problem; it should be investigated.

\section{Response:}

Project personnel agreed that the cited example demonstrates an error. It is not clear at this point whether this is an quantification, organizational, or clerical error. In any case, it is being investigated and represents a conservative versus a nonconservative error.

\section{Comment R2:}

At this point, uncertainty analysis has not been included. Plant-specific component failure data will be critical to the uncertainty analysis.

\section{Response:}

A full uncertainty analysis and discussion will be included in the Phase III report.

\section{Comment R3:}

As with importance analysis at the functional top event level, importance analysis at the component level can potentially yield interesting insights and should be considered for inclusion in the assessment. The same component failure can contribute to the failure of more than one top event. Importance analysis could indicate the relative importance of supporting systems.

In the N Reactor PSA, 32 basic events contributed to more than $99 \%$ of the risk when evaluated using the importance measure described in Vesely et al. (1983). 
Response:

The Phase III report will include additional importance analysis including importance at the component level.

Comment R4:

Many sequences end in less than total core damage, allowing the potential for the core damage frequency to be misinterpreted. Partial core damage states can be a useful tool in describing the results of the Level 1 PSA. The results of the $N$ Reactor Level 1 PSA were critically examined long before the Level $2 / 3$ results were published. It is important to include partial core damage states in final documentation.

Response:

Modification of the core hazard states is being performed in the Phase III effort as described in Comment E1. This is recognized as an important development. Only end states now classified as " $D$ " are being addressed. Those classified as " $V$ " are dominated by single tube rupture and are not further differentiated. Similarly, those accident sequences classified as " $A$ " are already catastrophic and involve the whole core, so they are not further differentiated.

Comment R5:

Recovery actions have not been included at this point. Inclusion should be considered because of the impact they potentially have on core damage frequency.

Response:

Recovery action will be addressed in only a limited fashion in Phase III as described in Comment 07.

\section{A.7 References}

Almenas, K., A. Kaliatka, and E. Uspuras. 1994. Ignalina RBMK-1500, A Source Book. Lithuanian Energy Institute, 3 Breslaujos, Lithuania.

Dougherty, E. M. Jr., and J. R. Fragola. 1988. Human Reliability Analysis. Science Applications International Corporation. John Wiley \& Sons, New York.

Hickman, J. W., et al. 1983. PRA Procedures Guide. NUREG/CR-2300, American Nuclear Society for the U.S. Nuclear Regulatory Commission, Washington, D.C. 
Sandia National Laboratories. 1989. Severe Accident Risks: An Assessment for Five U.S. Nuclear Power Plants. NUREG-1150, U.S. Nuclear Regulatory Commission, Washington, D.C.

Swain, A. D., and H. E. Guttman. 1983. Handbook of Human Reliability Analysis with Emphasis on Nuclear Power Plant Applications. NUREG/CR-1278, U.S. Nuclear Regulatory Commission, Washington, D.C.

Vesely, W. E., T. C. Davis, R. S. Denning, and N. Saltos. 1983. Measures of Risk Importance and Their Applications, NUREG/CR-3385, U. S. Nuclear Regulatory Commission, Washington, D. C.

Zentner, M. D., J. K. Atkinson, P. A. Carlson, G. A. Coles, E. E. Leitz, S. E. Lindberg, T. B. Powers, and J. E. Kelly. 1990. N Reactor Level 1 Probabilistic Risk Assessment: Final Report. WHC-EP-0322, Westinghouse Hanford Company, Richland, Washington. 
Appendix B

\section{Phase III Comments and Responses}




\section{Appendix B}

\section{Phase III Comments and Responses}

This appendix records comments and responses generated for Phase III of the Barselina project. Phase III is the final phase of the Level 1 PSA for the Ignalina, Unit 2 reactor performed by the Barselina staff. This record consists of PNL review comments and the corresponding responses given by the Barselina staff. It contains discussions pertinent to all issues raised during the PNL review of the Barselina project PSA.

\section{B.1 Overall Scope and Assumptions}

\section{Comment 01:}

The comparative analysis of the Ignalina and Barseback facilities is brief (Volume 5, Appendix A, Comparison of Ignalina and Barseback PSA, Section 5). This comparison is a stated project objective (Section 1) and is seen as a major thrust of the entire project. More comparative discussion would be appropriate and would serve to enhance project understanding. This section could generate insight into identified areas of risk that are of interest to Western reviewers. There are numerous comprehensive comparison charts that could be summarized to support this section. Examples could include insights such as 1) human errors important at Ignalina but not Barseback and 2) the justification for this. Consideration should be given to expanding the scope of the comparative analysis.

Response:

Discussion in Volume 5, Appendix A, Comparison of Ignalina to the Barseback Facility, will be improved after the Barseback plant has updated its PSA (specifically, extended the initiating event list). This is required for a more informative comparative analysis.

\section{Comment 02:}

No general plant description is provided in the PSA documentation. There are system descriptions in Volume 3 , Section 5, but these are independent discussions and are somewhat disconnected. Most significant is the limited information on the reactor core; this should be addressed as an independent section. It is difficult to get a clear understanding of the overall facility, including the system interfaces, from this material. A good technical description of the facility will enhance the readability of this report. Considering the RBMKs unique design, basic information such as the reactor core should be included. 
Response:

A separate document providing general plant technical descriptions will be developed. This effort will be separate from Phase IV activities. The Ignalina plant source book, according to the plant staff, contains some inaccuracies. The proposed documentation will append these inaccuracies and incorporate the plant system schematics and drawings used in the PSA.

Comment 03:

No importance analysis addressing either cutsets or basic events is reported in Phase III documentation. A stated project objective (Section 1.2.2) is the identification of systems, components, and human interactions important to safety. An importance analysis using the resulting cutsets provides an effective way to obtain this objective.

Material presented in Volume 2 addresses the dominant accident sequences and their contribution to the core damage frequency (for sequences pertaining to a specific initiator). This material also identifies the dominant cutsets and their contribution to the sequence in which they are contained. However, an importance analysis at the basic event level is not provided, and this can mask the significance of individual events connected with the total core damage frequency. For example, if a certain failure is found to have a modest contribution to a large number of cutsets, it may in turn have a very significant importance factor. We believe that such an analysis would indicate the importance of certain human errors that occur in numerous sequences.

The N Reactor PSA identified 32 basic events that contributed to more than $99 \%$ of the risk. This insight was identified through an importance measure described in Vesely et al. (1983). Consideration should be given to providing an importance analysis.

Response:

An importance analysis has been completed but was not included in the Phase III documentation. An improved importance analysis will be included in the Phase IV documentation.

\section{Comment 04:}

No quantitative uncertainty analysis is reported in Phase III documentation, although performance of uncertainty analysis is within the stated scope of this assessment (see Volume 1, Section 1.3). Quantification of uncertainty is an important issue due to the unique nature of the RBMK design and operating culture. Uncertainty will be an important asset of the comparative analysis to be conducted with the Barseback and other commercial plants. This issue has elevated significance due to the data and modeling limitations created by a fundamental lack of archived information. Quantification has been performed, addressing the effect of a 
proposed set of risk reduction measures reported in Volume 7, Appendix F, along with a brief qualitative discussion of uncertainty. However, a formal uncertainty analysis is not reported and should be considered for inclusion.

Response:

Probability distributions for the input data have been difficult to develop, but an uncertainty analysis is planned for Phase IV.

\section{Comment 05:}

Justification for assessing only the full-power operational modes is not found in Volume 1, Introduction and Scope Discussions; Volume 8, Limitation Discussions; or in Appendix G1-G3 (project scope discussions). Pre-Chernobyl PSAs typically assumed that the dominant risk was associated with full-power operation. This assumption has been challenged ever since the core melt accident at Chernobyl, Unit 4, which occurred at low power. The similarity of the Ignalina design to that of Chernobyl warrants consideration of other-than-full-power events by the Barselina Project.

\section{Response:}

The basis for selection and scope of initiating events is provided in Volume 1 , Section 3.1.3 (Initiating event analysis). A Chernobyl-type event is no longer credible due to changes in the operating culture and design at RBMK plants. This event can be categorized as an initiating event with a large fast reactivity insertion due to severe violation of operational limits. This scenario represents a type of event that is difficult, if not impossible, to cover in a PSA analysis. A PSA must, to a large extent, rely on the basis formulated by the deterministic safety analysis and the corresponding operational safety limits.

\section{B.2 Initiating Events}

\section{Comment 11:}

The MLD for reactivity initiated events is not shown. The discussion provided in Volume 4, Section 6.6.2.3 (which addresses the inclusion of all the elements) states that a screening analysis was carried out according to the structure in Figure 2 , but there is no Figure 2. If Figure 2 were provided it might show the MLD or some other screening structure. The inclusion of this figure could enhance the understanding of this assessment and support recognition of the scope and completeness.

Response:

This editorial comment is acknowledged; the figure containing the MLD is missing. 


\section{Comment 12:}

The discussion addressing the initiator $\mathrm{TS}_{\mathrm{FAS}}$ (Volume 1 Section 3.4.1, Transients and Reactivity Events) refers to these events as class R3:1 and R3:2. The discussion provided in Volume 4, Section 6.6.2.3, (Protection and Reactivity Control Initiating Event Screening Analysis) also refers to these classes (R3:3-13). These classes apparently have been taken out of the Initiating Event Screening Matrixes. This reader cannot follow the screening process in Section 6.6 but concludes that all reactivity events are assumed to be covered by $\mathrm{TS}_{\mathrm{AZ}}$ or $\mathrm{TS}_{\mathrm{FAS}}$.

\section{Response:}

This editorial comment is acknowledged. Identification and discussion of several initiator event category categories were moved from the initiating event screening tables to the common-cause initiator section of the report. This makes the screening process more complex and difficult to follow. It also recognized that the reactivity modeling is greatly simplified.

\section{Comment 13:}

The discussion in Volume 4, Section 6.8.4, Loss of Ventilation In Control Logic Rooms (as a common-cause initiator), states that this event was not analyzed and assumed to lead to manual shutdown or spurious SCRAM. Operational history reported on in Volume 3, Section 5.13.1.4 states that the ventilation for certain rooms was upgraded after environmental overheating failed Integrated Circuits (ICs) associated with the drive control blocks. There are apparently many ICs associated with the SCRAM actuation systems that appear to be sensitive to overheating. This effect could represent a common-cause event.

It is nonconservative to assume that common-cause failure of ICs always leads to a fail-safe condition. Failure of ICs may include unusual failure modes. Using solid state devices incorporated into the SCRAM logic needs further investigation. These devices are seen as atypical and warrant further thought. Recent operating history has shown that spurious relocation of the control rods has occurred due to environmental heating effects.

Response:

Integrated circuits are used in Swedish nuclear reactor plant SCRAM control systems without the noted concern. However, the comment is acknowledged and will be investigated.

\section{Comment 14:}

There is no explanation for or reference to the technical basis for the success criteria given in Volume 2, Section 4.4.1 involving reactor shutdown events. This inadequacy is again acknowledged in Volume 7 Section 4 . The local success 
criterion in Volume 2, Section 4.4.1 for the normal control rod includes the cryptic designation "not more than $Y$ adjacent rods." The local success criterion needs to be defined and justification provided.

\section{Response:}

Improved reactivity control system success criterion will be developed in Phase IV for both local and general reactivity transients.

Comment 15:

It is not clear whether the general reactivity success criterion bounds all possible reactivity insertion events. The initiating event table given in Volume 1, Section 3.6, Table " $X$ " does not include any events labeled as reactivity insertion events. It is not clear whether such events are covered by the automatic, manual, or fast SCRAM cases (TM, TS, and TSF). The success criteria.for modeling the Reactor Control and Protection system could potentially take these reactivity insertion events into account. Accordingly, the basic issue to be addressed involves the reactivity control margin for transients that also insert reactivity. The analysis reported in Volume 4, Section 6.6 implies that there are no reactivity events that challenge the success criteria of the shutdown systems (AZ-1 and FASS).

\section{Response:}

Success criterion for the reactivity control system is based on the worst-case basis (LOCA). Section 6.6.2.3 provides a qualitative discussion of how all other reactivity insertion events are insignificant or bounded by other sequences.

\section{Comment 16:}

The steam drum rupture is not considered an initiator. The screening analysis in Volume 1, Section 3, Enclosure 1 does not address failure of the drum separator as an initiating event. Previous project documentation (Phase II) stipulated that the drum separator rupture event was beyond design basis. It is presently assumed to be an extremely low probability event, which precluded inclusion. A description is needed to explain why this failure is insignificant. This review questions the frequency of this event and the understanding of its ultimate consequence. We note that Volume 7, Appendix E5 (listing of pipe parameters) designates the drum separator under a category characterized as "Beyond."

\section{Response:}

Section 2.5 .5 of the report addresses why steam drum rupture is not included in this assessment. This section states that a drum separator rupture has a failure frequency the same or a factor of ten less than the failure frequency of the main cooling pump (MCP) header (2E-5/yr). Probabilistic fracture mechanic calculations from the Research and Development Institute of Power Engineering (RDIPE) indicate 
that the failure frequency might be 1E-10/yr. It is acknowledged that this issue should be included in the sensitivity analysis, because it is a single failure of the reactor plant.

\section{Comment 17:}

In general, the technical basis for success criteria is still under development. The success criteria for various LOCAs are, compared with other reactors, very complex. Some of the criteria have been identified as uncertain. The discussion in Volume 7, Appendix F1, Section 5, Sensitivity to Model Limitations, states that a lot of deterministic calculations are missing. We further note that no Western-style SAR has been done. SARs normally supply information that support and provide a technical basis for PSA activities. In its absence, we agree that a lot of deterministic analysis is still required to increase confidence in the success criteria.

Although an SAR (NUSAR) was completed for the N Reactor, the concern about success criteria was an issue. For example, one of the final findings in the $N$ Reactor PRA involved a certain break location that might be problematic. This issue wasn't discovered until very late in the study and was found due to the intense RELAP 5 modeling supporting the PRA. N Reactor is unique among Western plants and; like the RBMK, could not always draw on other commercial plant experience. Because of this, uncertainty about success criteria remains an important issue. Consideration should be given to additional analysis and treatment to better define success criteria.

\section{Response:}

Any issues affecting success criteria important to the risk assessment are identified in the SAR. This effort will be addressed in Phase IV. Risk significant refinements will be made and quantified.

Comment I8:

The treatment of Process Control Failure Common Cause Initiators (discussed and analyzed in Volume 4, Section 6.6) is not extensive. Working from a candidate list of 24, rationale was given for excluding all but two initiator categories: 1) loss of pressure control for deaerator and 2) low-level or loss of coolant in channel cooling system. Three kinds of rationale are given: 1) insignificant when compared with other dependencies, 2) low frequency, and 3) could be considered to be covered by an another initiator category. These rationale were brief and qualitative and not always conclusive to this reviewer. For example, as described below, certain common-cause effects were not investigated.

- Loss of pressure in an ECCS pressurized tank was excluded as insignificant compared with other dependencies. This discussion considered that only one of three trains becomes unavailable. Common-cause failure of two or three trains was not considered. The consideration of common cause could 
be meaningful, because there is no automatic pressure regulation, only human operator monitoring. This supports the potential of the commoncause failure of two or more trains.

- The drum separator (DS) level control failure was excluded on the basis that it has a low-frequency occurrence. This justification is based on the interconnection of the drum separators so that the DS level control in both drum separators must fail to create a problem.

The concern for the treatment of the drum separator process control common-cause initiator involves two issues: 1) The possibility of a common-cause failure impacting the operation of critical DS level meters is not known. This is not addressed and might be important. The per-hour failure rate of this event when taken over the number of operating hours in a year could be significant. 2) The possibility of a transient occurring that requires more than one successful drum separator and corresponding level control warrants further investigation. The potential transient and loss of indication could challenge the ability to determine adequate core cooling. DS level indication and control is extremely important to operation and accident mitigation. A drum separator level failure initiating event may ultimately lead to the loss of a vital reactor critical safety function (core cooling).

\section{Response:}

In general, common mode failure effects related to common-cause initiators are indeed considered. The ECCS pressurized tanks are not an issue, because their failure does create a challenge (transient) in the plant. Common mode failures higher than combinations of two are considered to be too improbable and not significant.

\section{Comment 19:}

The area-events common-cause initiator analysis described in Volume 4, Section 6.9 is an enhancement of this study; however, it is limited to consideration of only those rooms where critical equipment is located. It is possible that halls and corridors, because they contain signal wire, power lines, and piping, might be as or more important than rooms. The N Reactor PSA determined that a fire in such a critical corridor was one of the dominant risk contributors leading to core damage. The N Reactor was constructed before implementation of the Nuclear Regulatory Commission-driven Appendix $\mathrm{R}$ considerations, which would have provided preconstruction resolution of this type of issue. The Ignalina assessment considers only rooms where end-user pieces of equipment are located. As with $\mathrm{N}$ Reactor, the Ignalina facility lacks many of those design, construction, quality assurance, operational, and maintenance protection factors that would mitigate the significance of some area-specific events. This determination heightens the significance placed on a scrutable assessment of these initiators. 
Response:

The main areas of power or signal routing are adjacent to the control room. The analysis for room dependencies involving active components (Section 6.3.9) has identified these rooms (D254 and A200) as important. This analysis will be integrated into the quantification during Phase $\mathrm{N}$.

\section{B.3 Event Tree Modeling}

\section{Comment E1:}

Table 1 at the end of Section 4.3.4 appears to be the "official". representation of the core hazard categories. The intended use of the more sophisticated schemes presented in Table 2 and Table $1 \mathrm{~A}$ is unclear. These more sophisticated schemes amplify the fact that there are more degrees of mechanistically possible core damage states. It is not clear that these other categories are intended to be addressed.

A broad range of core damage states is theoretically possible for the channel reactor design. For example, it is possible that fuel in $<90$ technological channels melts and within time can rupture their associated tubes (see Figure 46 of Appendix B9 for GDH rupture, where temperature continues to rise above $1200^{\circ} \mathrm{C} 900$ seconds into the accident). The reactor vessel cover would not necessarily lift under this delayed scenario, because the pressure relief system is venting pressure with decay heat, and reactor pressure is decreasing. Depending on system performance, different degrees of damage are possible. It might be possible to melt or partially melt fuel in 91 technological channels and not rupture the tube.

Response:

Table 2, Assignment of Hazard States, in Volume 2, Section 4.3.4 contains the core damage categories used in this assessment. It is acknowledged that the description in this section should clarify this fact.

\section{Comment E2:}

The event trees shown in Volume 9 do not show the expected failure frequency for any sequences that result in a loss of reactivity control except for top event, C2.3 (FASS Rod Slow Insertion). Sequence frequencies involving top event C2.3 are quite low (1E-9 to $1 \mathrm{E}-13)$. It is unclear whether other sequences frequencies are too small to report. In the manual and auto-SCRAM event trees, there are frequencies reported for other sequences; however, the ones shown do not involve the failure of the SCRAM system but rather subsequent core cooling failure. The expected frequencies for reactivity control failure need to be understood, justified, and treated consistently throughout the assessment. 
Response:

The expected sequence frequencies that involve loss of reactivity control are too low to be reported $(<1 E-11 / y r)$. This is acknowledged to be optimistic, and related fault tree models will be redone in Phase IV.

Comment E3:

In both the Ignalina 1-2 General Plant Description and Table 3.1 (Appendix A) the blowdown and cooling system (BCS) is shown to be an important heat removal system. This system does not appear to be addressed in the PSA. It appears to be the normal heat removal system for uncomplicated shutdowns. Some credit could potentially be taken for the BCS in certain accident scenarios. The event trees in Volume 2 for manual and auto-SCRAM (with all systems available) demand ECCS but not the BCS. Consideration should be given to the reassessment of the importance of the BCS within this assessment.

Response:

The BCS is a low-pressure system and cannot be used until the system temperature is down to about $180^{\circ} \mathrm{C}$ (about 10 hours after transient shutdown). Thus it is not useful in many accident cases. This system was considered earlier in the project, but not modeled in this study.

\section{Comment E4:}

The analysis reported in Volume 4, Section 6.10 (Availability of Systems after LOCA) does not appear to be used in quantification of the core damage frequency. This issue is addressed only as a sensitivity. The conclusions for Section 6.10 do not address pipe other than technological channels (TCs); however, Table 3.1 presents a respectable summary of dynamic effects and consequences for a number of LOCA locations. Table 3.1 acknowledges that certain pipe breaks could propagate into failure of neighboring equipment, structure, or pipe.

As noted in Volume 8, Section 5 (Sensitivity and Model Limitations) and Volume 8, Appendix G1, Section 5, the reviewer agrees with the need to address the issue of dynamic loads and their impact.

The issue of process tube rupture propagation is not specifically discussed as a sensitivity. The possible significance of this effect is great. If mechanistically possible, this event could be catastrophic to the safety of this design.

Consideration should be given to increasing the scope of the assessment to include treatment of system availability after a LOCA (in addition to flooding). The lack of protection provided through the design, construction, and operation of the Ignalina facility enhances the need, to understand the strength of the facility to sustain and respond to an event. 
Response:

The analysis in Volume 4, Section 6.10 regarding dynamic and secondary effects is not complete. Investigation of this issue represents significant work and will not be pursued in Phase IV unless the SAR provides information that indicates it should be incorporated into Phase IV risk quantification.

Comment E5:

The secondary effect of steam was not taken into account in this analysis. Steam is mentioned in parenthesis in Volume 4, Section 6.9 as an issue to be considered along with flooding. It is unclear how steam effects are accounted for in the flooding impact index determinations or the area event analysis sheets. Volume 8 , Appendix F1, Section 5.2 states that the qualification of electrical equipment for environmental conditions such as steam during an accident should be addressed (in the future).

Response:

The effect of steam was only considered as flooding source. Secondary effects (dynamic) are not integrated into quantification. If risk-significant issues related to secondary effects are raised in the SAR, they will be addressed in Phase IV.

\section{B.4 Fault Tree/System Modeling/Human Reliability Analysis}

\section{Comment F1:}

The potential impact of debris such as insulation or coveralls that could be carried into the ALS pools needs to be considered. This could potentially cause degraded ECCS flow or other related damage. Such an event is not modeled in the ECCS fault tree shown in Volume 10.

Response:

Plugging caused by debris washed in from recovered steam (during primary system blowdownl is not an issue. The situation at this reactor is different from other commercial reactors, because the ALS pools are above and relatively far away from the reactor block, making it unlikely that debris could be lifted into the ACL pools. The walls are steel and concrete without thermal insulation. Occupation is not allowed in this area during reactor operation.

\section{Comment F2:}

The Reactor Protection and Control System may not be adequately modeled. The fault tree in Volume 10 for the Reactor Protection and Control System does not show any consideration of common mode failures. The results discussion in 
Volume 1, Section 2.11.2, states that its conclusions are very much dependent on the assumption that the SCRAM function has a sufficiently high reliability. It goes on to state that other PSA studies have shown that a full or partial failure to SCRAM is an important contributor to risk. In this study, a failure to SCRAM does not contribute to the results at all. The issue is complicated by the lack of a boron injection or some other independent secondary reactivity control mechanism. With these additional considerations the reliability might actually be lower than average. Consideration should be given to increasing the scope and depth of the assessment addressing the control and protection system.

\section{Response:}

Lack of common-cause, dependency, and area event concerns needs to be addressed in fault tree modeling of the reactivity control system. This comment is acknowledged and will be addressed in Phase IV.

\section{Comment F3:}

Solid state logic for reactivity control in Western plants has been a concern due to the failure mode predictability. The design of the reactor control and protection 'system (CPS) relies extensively on solid state logic for detection and actuation. This is a sensitivity issue and warrants further investigation.

\section{Response:}

Swedish plants use integrated circuits in their reactivity control systems. Further investigation is warranted to identify the applicability of this concern to the Ignalina CPS.

\section{Comment F4:}

The Volume 4, Section 6 table of contents identifies the diesel generator data and common-cause impact vector analysis as found in BPR(93)61; however, the report is not included in the referenced section. It is unclear whether additional analysis is needed, has been, or will be done. Section 6.4 provides some common-cause impact vector analysis for Ignalina Nuclear Power Plants (INPP) diesel generators, but it is unclear whether this is the same work.

\section{Response:}

This analysis of alpha factors for diesel generators is actually included in the Phase III documentation and does not exist in a separate report.

\section{Comment F5:}

It is not clear from the fire analysis (Section 6.9, pp. 2, 3 and 4 and the area event analysis data sheets) that consideration was given to the impact of transient combustibles. Transient combustibles can significantly increase the fire loading of an 
area if not properly controlled. This issue is complicated by the lack of an aggressive program to control and limit transient combustibles within INPP. The $N$ Reactor PSA determined transient combustibles to be the dominant contributor to fire-initiated events. The assessment of transient combustibles represents an area of this assessment requiring additional attentions due the impact of the limited inplace administrative controls typically relied upon to preclude combustible intrusion.

Response:

Transient combustibles were not considered. The areas were evaluated for combustibles as they were viewed on the day of the walkdowns.

\section{Comment F6:}

Some Human Reliability Analysis (HRA) probabilities may be nonconservative. The HRA methodology described in Volume 6, Appendix C4 is innovative and nicely done but contains unstated assumptions. Key assumptions are made using a check list (see Table 3) to rate the attributes of an activity carried out by an operator. All attributes rated are assumed to carry an equal weight. Therefore, the attribute involving a lack of formal procedures can be put on equal footing with the attribute of personnel substituter rules. Western experience has shown that the lack of procedures could, in some cases, cause a severe accident or preclude mitigation. Using this approach, the effect of this disadvantage can only affect the failure probability by a small fraction $(14 \%)$. These effects are treated serially rather than in parallel.

Once evaluated, each attribute is quantified using a normal 1-to-5 scale in a linear fashion; it is then applied across a probability range on a log scale. Figure 1 in Volume 6, Appendix C1 illustrates this log-scale application issue very clearly. This methodology can induce significant uncertainty not mathematically warranted.

Response:

It is acknowledged that the weighing scheme used in the determination of error probabilities for human interactions may not be representative in certain cases. $A$ similar issue was uncovered when performing the area event impact factors. This issue deserves further consideration.

\section{Comment F7:}

It is not clear to this reviewer how the different approaches to obtaining HRA values were applied. Three different methods were discussed in Volume 6, Appendix C, and it is not clear which results were used for the various events. A NUREG/CR1278-style approach is described in Appendix C3; a new innovative Swedish approach is described in Appendix C4; and a simple scheme for manual shutdown is given in Appendix CO, Section 5.2. Both the Swedish and NUREG/CR-1278 
methods were applied to many events. The knowledge of the numbers that were used in the quantification and their basis for selection will enhance the use and understanding of this assessment.

Response:

It is acknowledged that the document needs further discussion of how the HRA was performed when error probabilities were calculated using more than one method. The RELCON method was the primary method used.

\section{Comment F8:}

It unclear if Table 4 in Appendix $\mathrm{CO}$ represents all the human interactions included as basic events (the other consideration being those considered as top events).

Response:

Human interaction events are included as basic events in the fault trees and events in the functional top event trees.

\section{Comment F9:}

Volume 1 refers to different classes of human interaction that have been addressed and modeled separately in the plant models: Type 1 being Operator inadvertently disables equipment..... to Type 5, Plant personnel fail to improvise or restore the operation of initially unavailable or failed equipment to terminate an accident. Volume 6, Appendix C (Human Reliability Analysis) does not refer to these types. This reviewer cannot determine that any Type 5 or 4 human interactions were included. Clarification will be required to understand the application of the different classes of human interaction.

\section{Response:}

The editorial comment about carrying HRA-type discussion into the HRA section in Volume 6, Appendix $C$ is acknowledged. In general, no Type 5 recovery events analysis has been done. Due to the dependence of the plant on manual human interaction for many initiators, several events that are similar to recovery events are included. In accordance with industry practice, few Type 4 (errors of commission) are included.

Comment F10:

Although a new system description (Primary Circuit) has been added to Volume 3 , no primary circuit fault tree modeling has been integrated into the quantification of core damage frequency. The last words in Volume 3, Section 5.17.3.2 are, "The model has no basic events. A discussion of the effects of certain component failures modes and...." The rest of this discussion is cut off. This section refers to functional top events such as U2.X and V2.X, which do not exist in Volume 9. It 
appears that combinations of check valve failures (as well as other blockage events) in interfacing ECCS headers are not accounted for. Consideration should be given to increasing the scope and consistency of primary circuit modeling.

Response:

The common-cause failure of various combinations of ECCS valves do appear in the functional event tree and show up in some of the accident sequences. Further modeling was deemed not to be advantageous.

Comment F11:

The discussion in Volume 4, Section 6.4.4 identifies those candidates for commoncause failure analysis. In addition to those listed, there are other sets of components that should be considered for common-cause failure, such as

- $\quad$ ECCS header check valves

- Equipment associated with short-term ECCS injection such as the pressurized tanks

- ECCS actuation relays (in the 1980 s there was a major concern at NRCregulated plants about mercury-wetted relays and common-cause failure)

- $\quad$ SW pumps

- Drum separator level instrumentation, such as level meters.

Additionally, this section does not identify diesel generators, ECCS pumps, and auxiliary feedwater system (AFWS) pumps, although they are identified on the attached table CCF-IG2.XLS. Consideration should be given to expanding the scope for the analysis of common-cause failures.

Response:

Actually, the system fault trees were used to generate candidates for common mode analysis. ECCS check valves are included; others were not inc/uded as significant, based on the system fault tree quantification.

\section{Comment F12:}

Instrument and control system modeling is inconsistent in scope and depth. Fault tree modeling for the actuation and control system (ACSS), the reactor control and protection system, and the main steam relief valves (MSRV) actuation system are quite detailed; however, other modeling is not. In general, the loss of power to many of these systems is not modeled.

The modeling for the deaerator pressure control is still primitive. It consists of the failure of the start signal to the emergency deaerator feeding pumps and includes only the failure of a level transmitter and a transducer. 
This review cannot detect that the drum separator level control was modeled in any fault tree. A basic event identified as, "Low Level in DS Due To Flow Regulation Errors," appears to cover the issue. The drum separator itself is not modeled.

Most importantly, loss of power is not modeled as a contributor to any control circuit failure. Because functional separation issues (created by common electrical busses) are a problem at Ignalina, this should be further explored. This review finds this issue to be an important undefined sensitivity that should be added to Volume 8. Appendix F.

Response:

It is acknowledged that the instrument and control modeling, in general, is inconsistent and lacking in detail. The deaerator and drum separator level controls are quite complex, and the modeling would require substantial work. It is believed that common-cause failures would dominate, and therefore only a dependency analysis is needed. Integration of the qualitative room dependency analysis into the quantification is needed in Phase $\mathrm{N}$.

\section{B.6 Data /Frequency and Probability Determination}

\section{Comment D1:}

The summary of estimated component reliability parameters for INPP-2 in Volume 7, Appendix E3, Table 1 contains some suspect determinations. The reported median values $(0.5$ quantiles) for components in which very few failures have been experienced is unrealistic. (For certain breakers: the mean $=2.1 \mathrm{E}-7$, the 0.95 quantile [percentile] $=8.16 \mathrm{E}-7$, and the median $=8.22 \mathrm{E}-17$ !) Section 3 acknowledges. that, in addressing the uncertainty of cases involving rare events, the median will differ somewhat from the mean. However, these parameters are not realistic, because they are essentially unbounded on the "zero side."

A detailed description of how these parameters were calculated is not provided. This determination is of interest because several failure rates involve a distribution arrived at based on "zero," or very few, occurrences. This reviewer believes that this approach can produce, for rare event estimation, distributions that are an artifact of the mathematics.

\section{Response:}

These probability distributions will be addressed in the uncertainty analysis to be done in Phase IV. The mean and 95th percentile can be fitted to gamma distribution. The 5th percentile side of the distribution should then be more realistic. 


\section{Comment D2:}

Section 6.4.3 states that plant-specific alpha factors are not available, which requires Western generic data to be extrapolated to the Ignalina case. The difference in operating culture and infrastructure could have a yet-to-be-determined effect on the alpha factors. This issue is further compounded by the degree of redundancy found at the facility and its impact on common-cause concerns. Consideration should be given to addressing this as a sensitivity noted in Volume 8 , Appendix F1, Sensitivity Analysis.

Response:

Results in Volume Section 2.8 (Sensitivity to Common-Cause Factor and Human Interaction) show that there is not a solid basis for putting the extra resource into developing plant-specific alpha factors. Figure 6 demonstrates that there is limited sensitivity in the results related to common cause.

Comment D3:

Volume 7, Appendix E9 describes in a general way how plant-specific, RBMK generic and world-wide data were all used to complete the study. A comparison study of failure rates, initiating events, and common-cause factors from these different sources might provide information about the general state of the data (as done in some specific cases such as LOCA frequencies). Consideration should be given to providing this comparative analysis.

Response:

It is acknowledged that this comparison might be useful, particularly with respect to the comparison with Barseback.

Comment D4:

The estimation of the LOCA frequency may be too low. Volume 7, Appendix E5 (LOCA Frequencies) describes a distributed zero estimation approach for determining LOCA frequencies for Ignalina. A total plant LOCA frequency is calculated based on the fact that the RBMK plants have not experienced a LOCA in their operating life. This frequency is then distributed among different LOCA categories.

This approach is based on the available data. However, the frequency is based on past performance, and performance might be expected to decrease as the plant ages. The much higher-than-average length of pipe, bends, and valves at the Ignalina plant should tend to exacerbate this aging problem. A special aging study was performed while performing the $\mathrm{N}$ Reactor PSA that addressed this aging problem and alleviated the concerns of critics. 
The small and large LOCA frequencies are much less than those for the N Reactor, even though both facilities employ a higher-than-average amount of pipe. Volume 7, Appendix E5, Section 4, Table 4 shows a frequency of $1 \mathrm{E}-3 / \mathrm{yr}$ for a small LOCA at INPP versus $2 \mathrm{E}-1 / \mathrm{yr}$ at $\mathrm{N}$ Reactor. The amount of small-diameter piping labout $100,000 \mathrm{~m}$ ) and valves (about 1800 ) is considerably higher than for other reactor designs and indicates that the small LOCA frequency should be higher than shown. Table 4 also shows a frequency of $1 E-4 / y r$ for large LOCA at INPP versus 6E-3/yr at N Reactor.

Volume 7, Appendix E5 reports the failure rates for pipes at $N$ Reactor to be $4.24 \mathrm{E}-$ 11 per hour per 30 meters $(\mathrm{m})$. This is true for large-diameter pipe but is incorrect for small-diameter pipe and should be reported as $4.24 \mathrm{E}-10 / \mathrm{hr} / 30 \mathrm{~m}$. Additionally, the N Reactor PSA reported that valve body failures contribute at a rate of $1.33 \mathrm{E}-$ $9 /$ failures/valve/hr. When these values are incorporated, a much greater small LOCA frequency is calculated.

If the small LOCA frequency is optimistic, the reported results are somewhat skewed. If the small LOCA frequency used in this study was increased by a factor of 100, the core damage frequency. associated with initiators S2-2 and S2-3 would be about $4 \mathrm{E}-6 / \mathrm{yr}$ apiece. They would tie for the seventh highest core damage sequence frequency reported in this document. Together, they would contribute about $20 \%$ to the total core damage frequency.

Response:

The LOCA frequency is based on the available RBMK-specific experience, which is about 120 operating years without an occurrence. This is the best information available. The distributed zero-estimation approach assumes that the ratios of large, medium, and small LOCA frequencies to the total are like those used in WASH1400 (Rasmussen 1975). Some pipe leaks have occurred, but these are not considered LOCAs and do not necessarily require plant shutdown.

It is noted that a major contributor to the N Reactor small LOCA frequency is spurious opening of relief valves. Spurious opening of relief valves has already been incorporated as an initiator frequency in this study.

\section{Comment D5:}

Conclusions about trends drawn from a zero and single occurrence analysis as described Volume 7, Section E6 may be overstated. Conclusions in Section 5 state that, in both cases, cited events demonstrate a decreasing trend of occurrence. This effect results from the generally increasing operating time logged per year. To conclude that the occurrence trend is decreasing seems overstated, particularly in the case where there were no events. Consideration should be given to the uncertainty involving rare event analysis and its impact on trend determination. 
Response:

The statement about trends based on zero or one event is acknowledged to be overstated. Consideration of a proper distribution for LOCAs, as well as for other initiators, when doing the uncertainty analysis is an important part of Phase $\mathrm{V}$. In some cases, Bayesian updating was done using only data from both INPPs for determination of the prior and from Unit 2 as the posterior. It is acknowledged that a better outcome would result if the prior distribution were based on a broader set of data.

Comment D6:

The initiator frequency for TS AE320 and TS AE209 levent initiators for rooms DS 209 and 320), reported in Volume 1, Section 2, Enclosure 1 is low (2E-4). The Ignalina Plant Description document shows that a serious fire event has already occurred in this area. Given this information, the initiating event frequency seems optimistic. The Berrys method for fire analysis described in Section 6.9 of Volume 4 may not account for significance of this plant-specific event. There may be a reason that the fire occurred at that location rather than another. Consideration should be given to the significance of this event and its impact on predicting future related events.

Response:

The fire event was the only one to occur for the whole plant. The calculated fire frequency for this room is based on the chance of having a fire in this particular location over some other location. No combustibles were found in this room. Using Berrys method, this room ranked only as a medium fire hazard and only a fraction of the total plant.

Comment D7:

Volume 6, Appendix CO, Section 3 states that both erroneously open and closed valves (failure to restore after test and maintenance) are given the failure probability of $1 \mathrm{E}-4$ and that no analysis has been made for these failure modes. This could be an optimistic value, because it is the lowest failure probability considered in the range of values using the RELCON method reported in Appendix C4. Documenting the basis for using this optimistic value will enhance understanding of the assessment. In many PSAs, a value of $1 \mathrm{E}-3$ or $1 \mathrm{E}-2$ is used; this value should be based on the number of independent verifications and chance for detection. The cultural impact of operational practices at Ignalina may warrant recognition relative to the conservatism used in value selection.

Response:

If the probability for failure to restore after test and maintenance were 1E-3, this event could become a risk-dominant event. Plant staff say a double signoff is needed to verify the valve position before startup. Plant staff have also indicated 
that, in many cases, the standby system is actually run to verify its in-service status. In any case, it is acknowledged that more justification in the text is appropriate.

\section{B.7 Quantification/Results}

\section{Comment R1:}

Although no uncertainty is reported for the quantified cutsets, uncertainty is expected to be high. For example, Section 4.30 .8 reports dominant cutsets for loss-of-offsite-power sequences, which are the most dominant sequences leading to core damage (according to Volume 2, Section 2). The failure of diesel is reported to be an important dominant event contributing to these accident sequences. The uncertainty parameters for diesel failures reported in Volume 7, Appendix 7 is quite large. The difference between the mean and 95th percentile is two orders of magnitude. The mean is close to the median, so the spread between the 5 th and 95 th percentile is likely to be fours order of magnitude. In short, this very important risk contributor has a very large uncertainty.

Response:

Again, uncertainty analysis will be included in the Phase IV effort.

\section{Comment R2:}

Many failure-of-reactivity-control sequences were not quantified. For example, in Volume 2 Section 4.5.6 (large LOCA, zone 1) it states that sequences 4,7 and 10 lead to an accident due to failure of the control rods; however, the frequency for these sequences is apparently not determined. They are not given in Figure 2 lonly the frequency for sequence No. 5 is given), and they are given in Section 4.5.8, which discusses dominant cutsets. This appears to be the case for all event trees. Another example is Section 4.21 (Automatic SCRAM All Systems Available). Section 4.21.6 states that, in Sequence 3 of Figure 2, the SCRAM fails due to failure of control rods, but the sequence frequency is not given in Figure 2 or in dominant cutset discussion.

\section{Response:}

Again, modeling of the reactivity control system will be improved in the Phase IV effort (including success criteria, common mode failures, dependencies, area events).

\section{Comment R3:}

Many sequence descriptions presented in Volume 2, 4.5 through 4.41 report that recovery actions are not taken onto account, although they potentially would have a 
significant effect on its associated accident sequence, particularly in conjunction with certain operator errors. It is unclear whether this is true for the entire analysis. Operator errors are important contributors to nearly all sequences, and nothing identified as recovery actions is ever presented. The Credibility and Qualification of Results section of Chapter 4 provides an inconsistent description of recovery events as they pertain to each event. Consideration of recovery actions, the consistency of application, and basis should be considered for inclusion within the assessment.

\section{Response:}

Modeling of recovery actions will be included in Phase IV as the plant follows recommendations to provide formal procedures to operators.

Comment R4:

No basic event importance analysis has been performed. For the sequence descriptions given in Volume 2, Section 4.5 through 4.41, the frequency of individual cutsets and basic events is not given in the listing provided for dominant cutsets. It could be instructive to see how important individual basic events are to the sequence frequency. An importance analysis could determine how often a basic event appears in different sequences. This, considered with the quantification of the basic event, is fundamental to understanding event significance. The review notes that a large number of certain human error events appear in many dominant sequences. The significance of these events may not be appreciated without a formal importance analysis. Additionally, the correlation between proposed engineering fixes and the importance of a component failure could be shown.

Response:

Again, importance analysis was performed but not reported. The report will be included in Phase IV documentation.

\section{Comment R5:}

The failure frequency associated with PCB3, Primary Circuit Blockage, is based on the value $1 \mathrm{E}-10 / \mathrm{hr}$ and is considered nonconservative. This is acknowledged in information provided in Volume 7, Appendix F2, Sensitivity Analysis of GDH Blockage, Section 5, which states that the calculated frequency for this event is a factor of 100 lower than the lowest blockage value that is used in the West for check valve and gate valve blockage. Accordingly, the frequency of this accident sequence could be $3 \mathrm{E}-3 / \mathrm{yr}$. Treating this issue in this manner tends to obscure its importance. The following table supports the claim about Western valves: 
Summary of Some U.S. Failure Frequencies

Related to Spurious Closure of a Valve

\begin{tabular}{|c|c|c|}
\hline Type & Frequency & Reference \\
\hline $\begin{array}{l}\text { Valves (motor-operated, manual) } \\
\text { Failure to remain open }\end{array}$ & $1 \mathrm{E}-7 / \mathrm{hr}$ & Sandia 1983 \\
\hline $\begin{array}{l}\text { Valves (all types) } \\
\text { Failure to remain open }\end{array}$ & $1.1 \mathrm{E}-7$ to $8.5 \mathrm{E}-6 / \mathrm{hr}$ & Zentner et al. 1990 \\
\hline $\begin{array}{l}\text { Motor-operated valve } \\
\text { Catastrophic plug }\end{array}$ & $5 \mathrm{E}-8 / \mathrm{hr}$ & Blanton and Eide 1984 \\
\hline $\begin{array}{l}\text { Motor-operated valve } \\
\text { Catastrophic spurious closing }\end{array}$ & $1.2 \mathrm{E}-7 / \mathrm{hr}$ & IEEE 1984 \\
\hline $\begin{array}{l}\text { Motor-operated valve } \\
\text { Spurious closing }\end{array}$ & $\begin{array}{l}20 \% \text { of all time- } \\
\text { dependent failures }\end{array}$ & RAC 1991 \\
\hline
\end{tabular}

Response:

This comment was acknowledged and will be considered.

Comment R6:

The accident frequency for certain sequences appears to be overpredicted. For example, in event tree S1-1T.C2 (medium LOCA zone 1, upstream of MCP valves), sequence No. 2 is shown as being 2.44E-7/yr. The discussion provided in Volume 2 , Section 4.9.8 states that this sequence is completely dominating and leads to core hazard state $\mathrm{AL}$, which corresponds to a very severe consequence. A success branch in this sequence occurs under top event V4.4 (manually isolate valves in the MCP lines within two minutes). The probability of failure, according to Volume 6 , Appendix $C$ is quite high, perhaps 1.0 . Consequently, the success of this event is quite low or zero. The accident sequence frequency of No. 2 in S1-1T.C2 does not appear to take this into account. When taken into account, this particular sequence is not dominating at all. This error could indicate a systematic quantification problem.

Response:

The miscalculation of sequence S1-1T.C2, No.2 is acknowledged. The inaccuracy is, of course, in the conservative direction. Any sequence that involves top event V4.4 (manually isolate valves in the MCP lines within two minutes) or V4.2 (manually isolate valves in the MCP lines within 10 minutes) may be subject to this kind of error, because the failure probability is 1.0 or near 1.0. This involves only a few large LOCA and medium LOCA sequences and is an overestimation. 


\section{B.8 References}

Blanton, C. H., and S. A. Eide. 1993. Savannah River Site Generic Data Base Development. WSRC-TR-93-262, Westinghouse Savannah River Company, Aiken, South Carolina.

IEEE Power Engineering Society. 1984. IEEE Guide to the Collection and Presentation of Electrical, Electronic, Sensing Component, and Mechanical Equipment Reliability Data for Nuclear-Power Generating Stations. STD-500-1984, The Institute of Electrical and Electronic Engineers, Inc., New York.

Rasmussen, N. C. 1975. Reactor Safety Study: An Assessment of Accident Risks in U.S. Commercial Power Plants. WASH-1400 (NUREG-75/104), Appendix VI, U.S. Nuclear Regulatory Commission, Washington, D.C.

Reliability Analysis Center (RAC). 1991. Failure Mode/Mechanism Distributions. Order No. FMD-91, Rome Laboratory, Griffiss Air Force Base, New York.

Sandia National Laboratories. 1983. Interim Reliability Evaluation Program Procedures Guide. NUREG/CR-2728, U.S. Nuclear Regulatory Commission, Washington, D.C.

Vesely, W. E., T. C. Davis, R. S. Denning, and N. Saltos. 1983. Measures of Risk Importance and Their Applications, NUREG/CR-3385, U. S. Nuclear Regulatory Commission, Washington, D. C.

Zentner, M. D., J. K. Atkinson, P. A. Carlson, G. A. Coles, E. E. Leitz, S. E. Lindberg, T. B. Powers, and J. E. Kelly. 1990. N Reactor Level 1 Probabilistic Risk Assessment: Final Report. WHC-EP-0322, Westinghouse Hanford Company, Richland, Washington. 


\section{Distribution}

No. of

Copies

\section{OFFSITE}

2 DOE/Office of Scientific and Technical Information

Eric Soderman

ES-Konsult $A B$

Arvid Momes vag 22

Box 3096

16103 Bromma

Stockholm, Sweden

Gunnar Johanson

Industriell Processukerhet AB

Svartviksslingan II

S-161-29 Bromma

Stockholm, Sweden

Jan H. Nistad

Behistedtsgatan II

Box 27106

S-102 52

Stockholm, Sweden
No. of

Copies

ONSITE

19 Pacific Northwest Laboratory

SQ Bennett

K7-90

SW Heaberlin

K8-34

SL McKay (8)

K8-31

GW McNair

K7-90

RL Moffitt

$\mathrm{K} 8-43$

MD Zentner

K8-37

Publishing Coordination

Technical Report Files (5)

5 Westinghouse Hanford Company

GA Coles (5)

H4-66 